University of Nebraska - Lincoln

DigitalCommons@University of Nebraska - Lincoln

April 2006

\title{
Toward a Formal Theory of Flexible Spatial Behavior: Geometric Category Biases Generalize Across Pointing and Verbal Response Types
}

John P. Spencer

University of lowa, john-spencer@uiowa.edu

Vanessa R. Simmering

University of lowa, vanessa-simmering@uiowa.edu

Anne R. Schutte

University of Nebraska-Lincoln, aschutte2@unl.edu

Follow this and additional works at: https://digitalcommons.unl.edu/psychfacpub

Part of the Psychiatry and Psychology Commons

Spencer, John P.; Simmering, Vanessa R.; and Schutte, Anne R., "Toward a Formal Theory of Flexible Spatial Behavior: Geometric Category Biases Generalize Across Pointing and Verbal Response Types" (2006). Faculty Publications, Department of Psychology. 10.

https://digitalcommons.unl.edu/psychfacpub/10

This Article is brought to you for free and open access by the Psychology, Department of at DigitalCommons@University of Nebraska - Lincoln. It has been accepted for inclusion in Faculty Publications, Department of Psychology by an authorized administrator of DigitalCommons@University of Nebraska - Lincoln. 
From: Journal of Experimental Psychology: Human Perception and Performance 32:2 (2006), pp. 473-490. Copyright 2006 by the American Psychological Association. DOI: 10.1037/0096-1523.32.2.473 Published by the American Psychological Association. Used by permission. "This article may not exactly replicate the final version published in the APA journal. It is not the copy of record."

\title{
Toward a Formal Theory of Flexible Spatial Behavior: Geometric Category Biases Generalize Across Pointing and Verbal Response Types
}

\author{
John P. Spencer and Vanessa R. Simmering \\ Department of Psychology, University of Iowa \\ Anne R. Schutte \\ Department of Psychology, University of Nebraska-Lincoln
}

\begin{abstract}
Three experiments tested whether geometric biases — biases away from perceived reference axes — reported in spatial recall tasks with pointing responses generalized to a recognition task that required a verbal response. Seven-yearolds and adults remembered the location of a dot within a rectangle and then either reproduced its location or verbally selected a matching choice dot from a set of colored options. Results demonstrated that geometric biases generalized to verbal responses; however, the spatial span of the choice set influenced performance as well. These data suggest that the same spatial memory process gives rise to both response types in this task. Simulations of a dynamic field model buttress this claim. More generally, these results challenge accounts that posit separate spatial systems for motor and verbal responses.
\end{abstract}

Keywords: spatial cognition, working memory, modeling, recognition, recall

People use information actively maintained in spatial working memory (SWM) in a seemingly effortless and flexible way. In particular, people can generate a variety of different responses based on location-related information held in working memory, including action responses (e.g., a reach toward the drawer to retrieve the keys) and verbal responses (e.g., "the keys are in the desk drawer"). Moreover, people can generate these different types of responses in the face of different task demands. For instance, adults can recall the locations of important objects in the absence of immediate perceptual cues (e.g., reaching for a coffee cup occluded by a stack of papers). They can also recognize or select a target location from an array of visible options (e.g., selecting the correct Styrofoam coffee cup on a table with several identical half-empty cups).

These examples raise several fundamental questions: What is the nature of the memory system (or systems) that underlies performance in these different situations, and what processes do people use to access SWM in the context of different response types and different response cues? Three literatures have produced initial answers to these questions; however, the answers differ in type (formal theories vs. verbal theories), content (one spatial memory system vs. two), and extent (whether they address developmental changes in spatial memory).

This research was supported by National Institute of Mental Health Grant RO1 MH62480 and National Science Foundation Grant BCS 00-91757 awarded to John P. Spencer. We thank the parents, children, and undergraduates who participated in this research. We also thank Gregor Schöner and Claudia Wilimzig for their valuable contributions to this article, Alycia Hund and Zabrina Marchant for all of their input and assistance with this study, and the many research assistants who helped with data collection. In addition, Nora Newcombe provided helpful comments on an earlier version of this article.

\section{Spatial Recall and Formal Theories of Spatial Memory}

One literature has focused on these questions by looking at the characteristics of spatial memory in recall tasks. In these tasks, participants are shown a single target location either in "empty" space (e.g., Mclntyre, Stratta, \& Lacquaniti, 1998; Soechting \& Flanders, 1989) or inside a geometrical figure (e.g., Huttenlocher, Hedges, \& Duncan, 1991; Huttenlocher, Newcombe, \& Sandberg, 1994; Laeng, Peters, \& McCabe, 1998; Werner \& Diedrichsen, 2002). The target is then removed, there is a short delay, and participants are asked to reproduce the target location, typically by pointing or placing an $\times$ at the remembered location.

One pervasive result across a number of studies of short-term recall is that people use visible reference axes - lines, edges, and symmetry axes - to help maintain location information in memory. Use of such axes provides a stable way to group or categorize objects. Nevertheless, there is a cost: When people are asked to reproduce the location of a target near a reference axis after a delay, they show geometric biases', that is, responses become systematically distorted away from the reference axis. For instance, Huttenlocher et al. (1994) asked 10-11-year-old children and adults to reproduce the locations of dots within a rectangular frame. Responses were biased away from the left and right edges of the frame and away from the midline symmetry axis (for related errors, see Engebretson \& Huttenlocher, 1996; Huttenlocher et al., 1991; Laeng et al., 1998; Nelson \& Chaiklin, 1980; Sandberg, Huttenlocher, \& Newcombe, 1996; Schiano \& Tversky, 1992; Tversky \& Schiano, 1989). It is important that recent data have shown that geometric biases emerge continuously during short-term delays. Spencer and Hund $(2002,2003)$ reported that geometric biases increased systematically across delays of 0-20 s, and Diedrichsen and colleagues (e.g., Werner \& Diedrichsen, 2002) found geometric biases at delays as short as $50 \mathrm{~ms}$. 
In addition to these empirical findings, the spatial recall literature has contributed two formal models of spatial memory - the category adjustment (CA) model (Huttenlocher et al., 1991) and the dynamic field theory (DFT; Schutte, Spencer, \& Schöner, 2003; Spencer \& Schöner, 2003). According to the CA model, people represent the fine-grained location of a target - that is, the direction and distance of the target from a reference locationand the category in which the target is located (Huttenlocher et al., 1991). Categories are formed by dividing space through the use of visible and mentally imposed axes. These category boundaries, along with the central, or prototypical, member of each category, are stored in memory. When asked to reproduce a target location, people combine their fine-grained and categorical representations on the basis of the certainty of the fine-grained information. Consequently, they produce errors away from reference axes and toward spatial prototypes because all locations within a region are weighted with the same prototype. For example, in the rectangledot task, participants divide the rectangle into two spatial categories with prototypes at the centers of the left and right halves. After a short delay, memory for an item in, for instance, the left category is weighted with the left prototype producing an error in this direction..

Although the CA model effectively captures the pattern of bias seen in spatial recall tasks, it does not specify the time-dependent processes that underlie the increase in geometric bias over delays (see Schutte \& Spencer, 2002; Spencer \& Hund, 2003; Werner \& Diedrichsen, 2002). The DFT captures these processes by using a neurally plausible network model that specifies how location-related activation is maintained in SWM during short-term delays (Spencer, Lipinski, \& Samuelson, in press; Spencer \& Schöner, 2003, 2006). Neurons in the DFT are spatially tuned such that neurons with similar "preferred" locations excite one another, whereas neurons tuned to very different preferred locations inhibit one another (for neurophysiological evidence of this type of interaction, see, e.g., Georgopoulos, Taira, \& Lukashin, 1993; Goldman-Rakic, 1995). As a result of this locally excitatory/ laterally inhibitory form of neural interaction, the model can sustain a localized "peak" of activation even in the absence of target-related input. This gives the DFT a robust form of working memory (see also Compte, Brunei, Goldman-Rakic, \& Wang, 2000; Renart, Song, \& Wang, 2003). It is important to note, however, that the DFT also captures how people use perceived frames of reference such as a midline symmetry axis (Spencer et al., in press). At the start of each trial, the model establishes "reference" peaks that are calibrated with perceived frames of reference in the task space. These reference peaks leave traces of activation in long-term memory. This allows the model to remember target-related information in a calibrated reference frame and to recall previous reference frames at a later time. The cost of this mechanism, however, is that reference-related information can bias information in working memory. In particular, self-sustaining peaks near a reference frame are stabilized by information in long-term memory, whereas peaks to the left or right of a reference frame are repelled from the frame because of strong laterally inhibitory interactions.

As this survey of the CA and DFT models demonstrates, both models effectively capture participants' geometric biases in spatial recall tasks. But how general are these models? Can they account for behavior in different tasks, tasks that probe memory in different ways or require a different response type? And how pervasive are geometric biases? Do they emerge only in artificial recall tasks when people must point to a location in empty space? These questions remain unanswered. As such, it is not clear whether these models provide a robust framework for examining the flexibility of spatial cognition.

\section{Sensorimotor and Verbal Response Types: Two Spatial Memory Systems?}

A second literature has examined the nature of the spatial memory system underlying flexible performance across contexts by comparing response errors when participants generate different response types, most notably, pointing versus verbal responses (e.g., Bridgeman, Gemmer, Forsman, \& Huemer, 2000; Brungart, Rabinowitz, \& Durlach, 2000). Here, there is some consensus that different response types tap different spatial memory systems: a "sensorimotor" system that encodes locations in the service of motor actions and a "cognitive" system that encodes locations in the service of verbal responses (Bridgeman, 1999; Bridgeman et al., 2000; Brungart et al., 2000). For instance, Brungart et al. (2000) asked adults to reconstruct a remembered location immediately after target presentation by using one of four response types - direct pointing or three types of verbal response. Pointing responses were the most accurate, yet unlike the verbal response types, such responses were sensitive to response delays (see also Spencer \& Hund, 2002; Werner \& Diedrichsen, 2002). Brungart et al. concluded that the motor system relies on a location memory that degrades whereas the cognitive system relies on a stable verbal report formed at target presentation (see Bridgeman et al., 2000; Bridgeman, Kirch, \& Sperling, 1981; Bridgeman, Peery, \& Anand, 1997; Proffitt, Bhalla, Gossweiler, \& Midgett, 1995).

Results from Crawford, Regier, and Huttenlocher (2000) also suggest that different representational systems underlie sensorimotor and verbal response types. When adults were asked to apply a spatial preposition (e.g., above) to a display depicting a target and a referent object, their judgments were most accurate along the cardinal axes of the display (vertical and horizontal). By contrast, pointing responses after a delay showed geometric biases away from the cardinal axes and toward diagonal axes. These researchers concluded that the representational systems that underlie verbal and pointing responses rely on different "prototypical" axes in the task space (for an alternative interpretation of these results, see Spencer et al., in press).

The proposal that different memory systems underlie sensorimotor and verbal response types makes sense given the demands placed on memory by these two response types. Pointing responses must be guided by continuous, metric spatial information, whereas verbal responses are categorical, coarse, and symbolic in nature (Huttenlocher et al., 1991; Jackendoff, 1996; Talmy, 1983). Nevertheless, this view of two representational formats raises a fundamental challenge: How do people effortlessly and flexibly generate different response types - in some cases, combining elements of both systems at very short response times (see Bridgeman et al., 2000)_-given the apparently incompatible nature of these spatial codes? Answers to this question require formal models that specify the interface or transduction process that integrates these codes; however, there are currently no models that specify such details (although see Jackendoff, 1996, for ideas in this direction), and 
neither the CA model nor the DFT has been extended to address performance in verbal tasks.

\section{Developmental Changes in Spatial Memory}

The two literatures discussed above yield unique insights into the flexible spatial performance of adults, insights that pose tough challenges for theories of spatial memory. A third literature presents yet another challenge: Spatial memory theories must explain how effortless, flexible spatial performance develops over time. At face value, the development of sensorimotor and verbal spatial abilities seems to be a case of discontinuous developmentsensorimotor spatial abilities emerge in infancy and show rapid change thereafter (e.g., Acredolo, 1985; Newcombe, Huttenlocher, \& Learmonth, 1999; Piaget, 1954), whereas verbal spatial abilities emerge much later, reaching proficiency by 5 to 7 years of age (e.g., Craton, Elicker, Plumert, \& Pick, 1990; Hermer-Vazquez, Spelke, \& Katsnelson, 1999; Plumert, Ewert, \& Spear, 1995; Plumert \& Nichols-Whitehead, 1996). Consistent with this discontinuous view, Hermer-Vazquez, Moffet, and Munkholm (2001) showed that 5- to 7-year-old children encode spatial relations in new ways once they become proficient at using spatial language. In particular, spatial language helps children combine geometric and nongeometric, featural information following a disorientation procedure that disrupts children's ability to use dead-reckoning to find a hidden object. Without spatial language proficiency, children rely solely on geometric cues in this task. Additional evidence from Hermer-Vazquez et al. (1999) has shown that spatial language plays an important role in adults' flexible integration of geometric and nongeometric information as well. When adults were required to engage in a verbal shadowing task that blocked their use of spatial language, they relied solely on geometric information to find a hidden object after being disoriented. This was not the case, however, when adults engaged in nonverbal shadowing of a continuous rhythm. In this case, adults combined geometric and nongeometric cues.

Although data from Hermer-Vazquez et al. $(1999,2001)$ are consistent with the discontinuous view, other data suggest that sensorimotor and linguistic abilities coevolve in early development. Learmonth, Newcombe, and Huttenlocher (2001) showed that 17to 24-month-olds can use nongeometric information to disambiguate a hiding location in the disorientation task developed by Hermer and Spelke (1994, 1996), provided that the task space is large enough and the nongeometric cues (i.e., landmarks) are salient and spatially stable. Additionally, cross-linguistic evidence has shown that infants learn to selectively attend to spatial distinctions made in their native language as they learn language. Hespos and Spelke (2004) showed that, at 5 months, infants from English-speaking households were sensitive to the tight- versus loose-fitting containment relations distinguished in Korean. By 18 to 24 months, however, children's attention to spatial relations begins to correspond to distinctions made in their native language (Choi, McDonough, Bowerman, \& Mandler, 1999). Considered together, results from these studies suggest a more continuous, coevolving view of the development of sensorimotor and verbal spatial abilities.

This continuity theme has also been echoed in the spatial recall literature. Recent evidence has suggested that two types of spatial recall biases-A-not-B-type biases and geometric biases- develop via continuous changes in process over development (Schutte, 2004; Schutte et al., 2003; Spencer \& Hund, 2003). For instance. Spencer and colleagues (Schutte et al., 2003; Spencer \& Schutte, 2004; Spencer, Smith, \& Thelen, 2001) have shown that, following repeated hiding and finding events at an " $\mathrm{A}$ " location, 2-to 6-year-old children show biases toward A when searching for a hidden object at a nearby "B" location. This bias is similar to the Piagetian A-not-B error made by 8- to 10-monthold infants (Piaget, 1954; Smith, Thelen, Titzer, \& McLin, 1999; Thelen, Schöner, Scheier, \& Smith, 2001). It is important to note that Schutte et al. (2003) quantitatively fit changes in such biases by systematically narrowing and strengthening the local excitation/ lateral inhibition function that governs neural interaction in the DFT. This was accomplished via continuous change in a single model parameter over development (for related efforts to capture developmental changes in geometric biases with continuous changes in parameters of the DFT, see Schutte, 2004; Spencer \& Hund, 2003).

\section{Overview of the Present Study}

Considered together, the three literatures discussed above raise fundamental questions about the nature and development of the spatial system (or systems) that underlies the generation of sensorimotor and verbal responses in different spatial tasks. The spatial recall literature offers two formal theories of spatial memory and key insights about continuous changes in memory processes over development; however, it is unclear whether these models generalize beyond sensorimotor responses in recall tasks. The two-systems literature offers a broader view of spatial performance but no formal theories to explain how a two-systems view can produce the unique characteristics of pointing and verbal response types in some tasks yet fast, efficient integration in others. The developmental literature echoes these themes. There is evidence of integration: Sensorimotor and linguistic spatial abilities appear to coevolve in early development. Yet there is also evidence that verbal abilities make a unique contribution to children's spatial abilities.

Thus, a central challenge is to understand how the balance between integration and uniqueness is achieved, that is, how a spatial memory system can flexibly integrate sensorimotor and verbal response types yet still produce behavioral signatures unique to each type. We contend that formal models can play an important role in addressing this challenge by highlighting the limitations of current views. For instance, formal models of a two-systems view must deal head-on with the problem of incompatible spatial codes if they are to engage in realistic, real-time - behavior. By contrast, formal models that pose an integrated-systems view must explain how behavior in the same task can differ when a sensorimotor versus a verbal response is required.

In this study, we took a first step toward expanding the role of formal theory in the spatial memory literature by asking whether the processes at work in the CA and DFT models generalize to tasks in which participants must make a verbal, recognitionbased response. In particular, we asked whether one type of biasgeometric bias - observed in spatial recall tasks with pointing responses generalizes to a recognition task with a verbal response. In addition, we asked whether such generalization depends on linguistic expertise by examining the performance of adults and 
7-year-old children. We selected 7-year-olds because previous research has shown that 7 years is the youngest age at which children begin to subdivide geometric figures along single spatial dimensions (Sandberg et al., 1996). It is important to note that this age is also within the range examined by Hermer-Vazquez et al. (2001). If geometric biases generalize to verbal recognition tasks, it suggests that the processes that produce the bias - processes formalized in spatial recall models - might be general as well. Results from three experiments suggest that this is the case but in a nontrivial sense: Geometric biases occur in both types of tasks but only in particular situations for each age group. Thus, in the General Discussion section, we consider whether these models can capture both the integration and the uniqueness of these response and task types. This sets the stage for a more general, processbased account of SWM.

\section{Experiment 1}

In Experiment 1, we used a modified version of the rectangledot task described previously. On each trial, 7-year-old and adult participants were shown a target dot within a rectangle followed by a short memory delay. After the delay, participants were cued to make either a sensorimotor response - to draw an $\times$ at the remembered location - or a verbal "choice" response. On these choice trials, participants were shown a set of colored dots, one of which was in the original target location. They were instructed to say the color of the dot that matched the target. Thus, some trials required participants to recall the target location and generate a motor response; other trials probed memory with a recognition or choice set (colored dots) and required a verbal response. It is important to note that participants did not know which response and task type would be cued at the end of each trial. Consequently, the task space, remembered locations, and memory delays were identical across tasks.

If geometric biases are unique to sensorimotor responses in spatial recall tasks, participants' responses on the draw trials should replicate the pattern of error reported by Huttenlocher et al. (1994); however, this pattern of error should not be observed on choice trials. Indeed, on the basis of previous studies showing that verbal responses remain stable during short-term delays (e.g., Bridgeman et al., 1997; Brungart et al., 2000) and that 7-year-olds are proficient at spatial language (Hermer-Vazquez et al., 2001), we expected participants to be relatively accurate in this task. It is also possible, however, that participants will show geometric biases on the choice task. This would demonstrate that this class of bias is not unique to a sensorimotor spatial system, suggesting that the processes formalized in the CA and DFT models extend to verbal responses in recognition tasks as well.

\section{Method}

Participants. Participants were 30 seven-year-olds $(M$ age $=7.52$ years, $S D=0.73$ months) and 30 adults $(M$ age $=19.27$ years, $S D=9.52$ months). Two additional children and 1 additional adult participated, but data from these participants were not included in the final analyses because of experimenter error. Potential child participants were drawn from a database at a large midwestern university; their names were originally obtained from birth records in the area. Parents of these children were contacted by letter and then by a follow-up phone call. Children received gifts worth approximately $\$ 3-\$ 4$ for participating. Adults were recruited through an introductory psychology course and received research participation credit. Each group consisted of roughly half male and half female participants.

Apparatus. Participants sat across from the experimenter at a long rectangular table (approximately $55 \mathrm{~cm}$ tall, measuring $165 \mathrm{~cm} \times 53 \mathrm{~cm}$ on top; see Figure 1). For most of the sessions with children, a parent chose to accompany the child for the session; in these cases, the parent sat in a chair to the left and behind the child, out of the child's field of vision. In front of the participant, $9.5 \mathrm{~cm}$ from the edge of the table, was a small frame $(21.6 \mathrm{~cm} \times 5.6 \mathrm{~cm}$ along the outside edges $)$ in which stimulus cards were presented. To the experimenter's left were an opaque stimulus card box and a button. When pushed, the button helped raise the stimulus cards out of the frame. To eliminate the possibility that other features of the room might be used as landmarks, a white canvas curtain surrounded the experimental area from floor to ceiling. The dimensions of the space within the curtain were roughly $2.4 \mathrm{~m} \times 2.1 \mathrm{~m}$.

Five types of stimulus cards were used: target cards, delay cards, draw response cards, choice response cards, and feedback cards. All cards measured $20 \mathrm{~cm} \times 4 \mathrm{~cm}$, were free of extraneous marks, and were white cardstock, with the exception of feedback cards, which were transparent plastic. Target cards displayed a gray dot, $1 \mathrm{~mm}$ in diameter, at a target location. Both delay and draw response cards were blank, though delay cards were made of heavier cardstock to ensure that targets could not be seen through them. Choice response cards showed five differently colored dots, all $1 \mathrm{~mm}$ in diameter, spaced $5 \mathrm{~mm}$ from each other. From left to right, the dots were colored black, green, orange, blue, and red. The location of the five dots varied from trial to trial such that one dot was in the target location on each trial. Feedback cards contained a circle, $3 \mathrm{~mm}$ in diameter, centered at the target location. When laid over a response card, the circle indicated the target location for a given trial.

Procedure. Each session began with a demonstration to ensure that the participant understood the task. Figure 2 shows the sequence of card presentations during this trial. The experimenter presented the target card in the frame and asked the participant to remember the location. Then the experimenter covered the target card with the delay card and instructed the participant to look up from the frame while counting aloud to 5 (for children) or 10 (for adults). After the delay, the experimenter sequentially presented both a choice response card and a draw response card; the order of the two response types was randomized across participants. When the

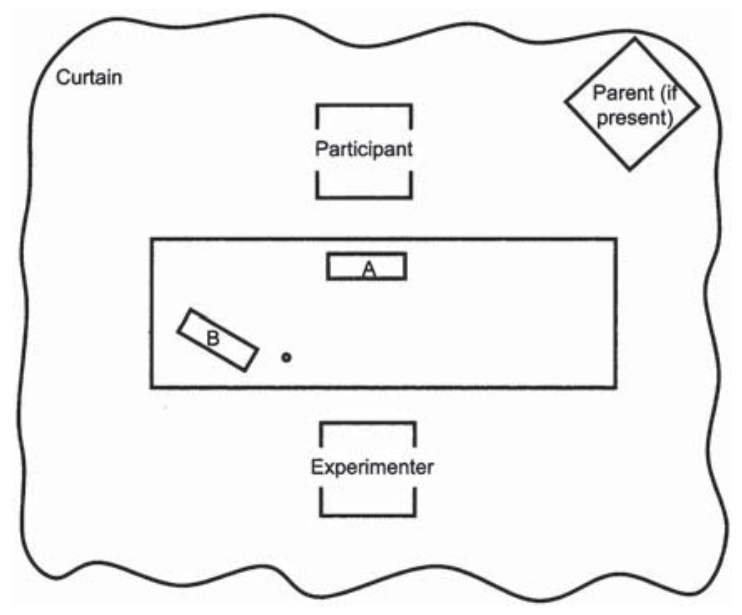

Figure 1. Setup of the experimental room. The experimenter presented stimulus cards (stored in the container at B) in the frame in front of the participant (see A). The curtain around the room eliminated potential landmarks. When present for children's sessions, parents sat in the comer of the room, out of the view of the children. 
1. Target Presentation

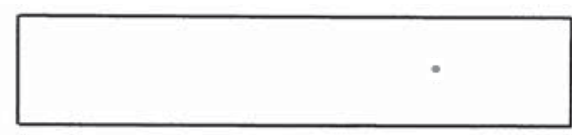

2. Delay ( $5 \mathrm{~s}$ for children, $10 \mathrm{~s}$ for adults)

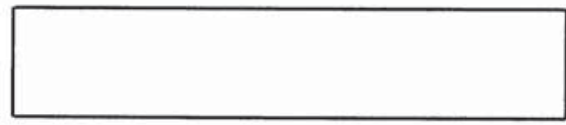

3. Choice Response Card

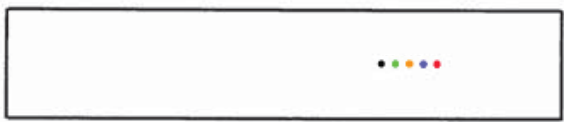

OR

Draw Response Card

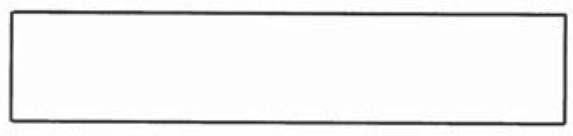

4. Feedback

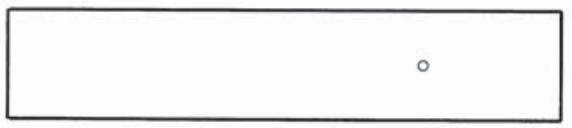

Figure 2. Event sequence for each trial. Participants were shown only one type of response card (i.e., choice or draw) on nondemonstration trials.

choice response card was presented, participants were asked to say the color of the dot that was in the same location as the target dot. The experimenter then repeated aloud and recorded the color the participant named. Next, participants were asked to name all of the colors from left to right, to ensure that they were discernible (and that the participants were not color-blind). When the draw response card was presented, participants were asked to draw an $\times$ where they remembered the target dot being. In the final step of the card sequence, the experimenter placed the feedback card over the response card and praised the participant's performance. Before proceeding, the experimenter asked whether the participant understood the task. If the participant did not understand the trial, the demonstration was repeated. Once the participant understood the task, the experimenter began the practice trials.

Each participant completed 4 practice trials, presented in random order. The sequence of events on these trials was similar to that of the demonstration, but only one type of response card was presented on each trial (note that feedback was given on every trial). Two of the practice trials were choice trials, and 2 were draw trials. After the 4 practice trials, the participant completed 26 test trials and 4 control trials (see the Experimental design section), presented in random order. The sequence of events in these trials was the same as for the practice trials. If a child participant appeared to be distracted during the session, a break was offered, though most children did not need breaks. Adult participants and parents were debriefed at the end of the session.

Experimental design. Thirteen locations were used as targets for the test trials. From the left edge of the card, the targets were at 12.5 -mm intervals beginning at $25 \mathrm{~mm}$ (ending at $175 \mathrm{~mm}$ ) and centered vertically on the card (see Figure 2). These locations were a subset of those used by Huttenlocher et al. (1994). ${ }^{1}$ Each target location was used once for a choice trial and once for a draw trial. Different locations were used for demonstration, practice, and control trials. For these trials, target locations were randomly selected from the 11 locations in between $(6.25 \mathrm{~mm})$ the target locations; these locations were not repeated.

For choice response cards, we randomly selected which colored dot would match the target for that trial. To allow participants to err in either direction on choice test trials, the dot that matched the target was selected from the three central dots rather than from either of the end dots. Given that this might cause participants to adopt a strategy in which they avoided the two end choices (because these choices were never correct on the test trials), we included control trials in which the end dots always matched the target. The end choices were also randomly selected as correct responses for some of the practice trials. Overall, the end choices were correct on approximately $25 \%$ of the choice trials.

Method of analysis. To compare performance across locations and response types, responses were coded as errors from the target. Draw responses were measured from the target location to the center of the $x$ drawn by the participant. Leftward errors were assigned negative values, and rightward errors were assigned positive values. Choice responses were calculated by measuring the distance between the dot that matched the target and the dot selected by the participant (leftward errors were negative). Thus, if the center dot (orange) matched the target and the participant selected the dot farthest to the left (black), the resultant error would be -10 $\mathrm{mm}$. Note that because the end choices never matched the target on test trials, the largest possible error was $\pm 15 \mathrm{~mm}$. Control and practice trials were not included in any analyses.

Given that choice response errors were categorical $( \pm 15 \mathrm{~mm}, \pm 10 \mathrm{~mm}$, $\pm 5 \mathrm{~mm}$, or $0 \mathrm{~mm}$ ) whereas draw response errors were continuous, draw errors were converted to categorical values. Specifically, values were rounded to the nearest $5 \mathrm{~mm}$ and truncated to $\pm 15 \mathrm{~mm}$. Statistical analyses revealed no significant differences between continuous and categorical draw errors. Thus, the categorical draw errors were used in all analyses.

\section{Results}

Figure 3 shows mean errors for both response types separately for children (Figure 3A) and adults (Figure 3B). In general, errors were smallest near the center of the rectangle (100-mm location) and were biased away from the center and the left and right edges. For instance, at $75 \mathrm{~mm}$, participants made relatively large errors to the left (negative values), and errors decreased as locations approached $100 \mathrm{~mm}$. Conversely, beyond $100 \mathrm{~mm}$, errors were rightward (positive values) and increased as they approached $125 \mathrm{~mm}$. Finally, adults' errors were generally smaller than 7-year-olds' errors despite the fact that adults had to remember the target location for a longer delay (10 s vs. $5 \mathrm{~s}$ for the 7-year-olds), and both age groups showed smaller errors on choice response trials than draw response trials.

To analyze these data, we conducted a three-way analysis of variance (ANOVA) with response type (choice or draw) and location $(25-175 \mathrm{~mm})$ as within-subject factors and age (7 years or adult) as a between-subjects factor. This analysis revealed significant main effects of location, Wilks's $\Lambda=.23, F(12,47)=12.80$, $p<.001$, and response type, Wilks's $\Lambda=.86, F(1,58)=9.54, p$ $<.01$. Additionally, there was a significant Location $\times$ Age interaction, Wilks's $\Lambda=.62, F(12,47)=2.43, p<.05$; a significant Response Type $\times$ Location interaction, Wilks's $\Lambda=.38, F(12$, $47)=6.53, p<.001$; and a significant Response Type $\times$ Location $\times$ Age interaction, Wilks's $\Lambda=.60, F(12,47)=2.70, p<.01$.

To explore these results further, we separately conducted simple effects tests for the adults and the 7-year-olds. Tests of simple effects for the children showed a significant main effect of location, $F(12,348)=9.06, p<.001$, and a significant Response Type $\times$ Location interaction, $F(12,348)=4.49, p<.001$.

${ }^{1}$ Huttenlocher et al. (1994) also used locations $12.5 \mathrm{~mm}$ and $187.5 \mathrm{~mm}$ from the left edge of the card. These locations were not used as targets here because they did not allow enough room for the choices presented on the choice response cards. 
Additional tests of simple effects revealed a significant effect of location for both choice responses, $F(12,348)=3.35, p<.001$, and draw responses, $F(12,348)=8.51, p<.001$. Thus, the positive and negative deviations across locations apparent in Figure $3 \mathrm{~A}$ for both choice and draw responses were statistically reliable. Furthermore, there were significant differences in error across response type at five locations: $25 \mathrm{~mm}, F(1,29)=17.91, p<.001$; $62.5 \mathrm{~mm}, F(1,29)=4.28, \mathrm{p}<.05 ; 75 \mathrm{~mm}, F(1,29)=9.26, p<$ $.01 ; 87.5 \mathrm{~mm}, F(1,29)=5.01, p<.05$; and $175 \mathrm{~mm}, F(1,29)=$ 15.03, $p<.001$ (see locations marked with arrows in Figure 3A). At all of these locations, draw errors were significantly larger than choice errors.

Simple effects tests for adults showed significant main effects of both response type, $F(1,29)=10.59, p<.01$, and location, $F(12$,
$348)=5.61, p<.001$, as well as a significant Response Type $\times$ Location interaction, $F(12,348)=2.88, \mathrm{p}<.01$. Additional tests of simple effects revealed a significant effect of location for both choice responses, $F(12,348)=2.64, p<.01$, and draw responses, $F(12,348)=5.56, p<.001$. As with the 7-year-olds, the positive and negative deviations across locations apparent in Figure 3B for both choice and draw responses were statistically reliable. Moreover, there were significant effects of response type at four locations: $50 \mathrm{~mm}, F(1,29)=7.28, p<.05 ; 87.5 \mathrm{~mm}, F(1,29)=10.76$, $p<.01 ; 150 \mathrm{~mm}, F(1,29)=12.43, p<.01$; and $175 \mathrm{~mm}, F(1,29)$ $=14.43, p<.01$ (see locations marked with arrows in Figure 3B). As with the 7-year-olds, draw errors were larger than choice errors for three of these locations. The difference at $150 \mathrm{~mm}$ was caused by errors in opposite directions across response types.
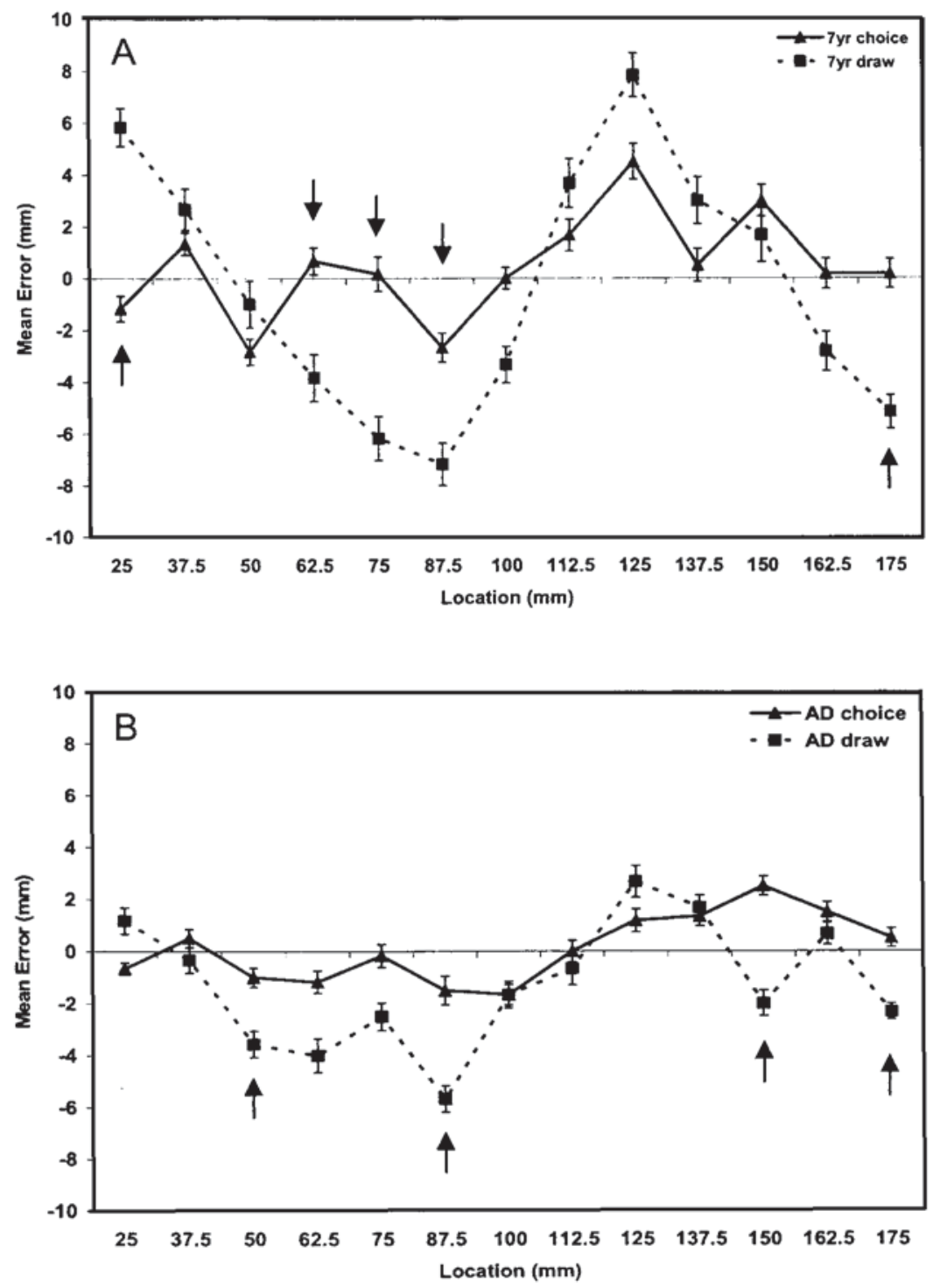

Figure 3. Mean choice and draw errors for (A) 7-year-olds (7yr) and (B) adults (AD) across the 13 target locations in Experiment 1. Positive values indicate rightward errors, and negative values indicate leftward errors. Arrows indicate locations at which response types differed significantly. Errors bars reflect plus or minus one standard error. 
Polynomial curve-fitting. In general, our data followed the same sinusoidal trend reported by Huttenlocher et al. (1994). To verify this result, we fit polynomial curves to the data from each response type and each age group separately (see also Huttenlocher et al., 1994). Table 1 shows multiple correlation squared $\left(R^{2}\right)$ values for the fits of quintic, cubic, and linear functions separately for each age and response type as well as significant differences in the fits of these curves. As with data from Huttenlocher et al. (1994), we found the best fit for children's draw responses was obtained by a quintic function, although the fit of the cubic function was also quite good. ${ }^{2}$ For adults' draw data, both quintic and cubic functions fit well, although only the fit of the cubic function was significantly better than chance (the fit of the quintic function was marginal). The cubic function also fit significantly better than a linear function. Thus, both sets of curve fits for the draw responses showed sinusoidal patterns similar to those reported by Huttenlocher et al. (1994). The difference in fits between the age groups likely reflects adults' more accurate responses, particularly at the edges of the stimulus set, which would affect the fit of a quintic function.

Because a verbal, recognition-based response has not been examined in this type of task, it is important to investigate whether these responses show the same sinusoidal pattern. If so, it would suggest that similar processes underlie sensorimotor, recall-based and verbal, recognition-based spatial memory errors. This was not the case for children's choice responses. As can be seen in Table 1 , there were no significant effects among the fits of quintic, cubic, and linear functions. Thus, there may be different processes underlying the generation of verbal versus pointing responses at 7 years of age. For adults' choice data, a quintic function fit significantly better than a linear function, and all three functions fit adults' choice data significantly better than chance. This finding suggests that, although adults were significantly more accurate in the choice task, similar processes underlie the generation of both types of response for this age group.

According to the CA and DFT models, the sinusoidal pattern evident in participants' responses is caused by systematic distortions in memory that reach their peak near locations of 25,75 , 125 , and $175 \mathrm{~mm}$ (the inflection points of the polynomial curves). This allows us to bring together results from the ANOVA and curve fits. Specifically, the locations at which response types differed (see Figure 3) were generally near the inflection points of the polynomial curves for both children and adults.

Table 1

\section{$R^{2}$ Values of Polynomial Curve Fits in Experiment 1}

$\begin{array}{llll}\text { Response } & \text { Quintic function Cubic function Linear function } \\ \text { 7-year-olds' draw } & .912^{\mathrm{a}}> & .727^{\mathrm{a}}> & .011 \\ \text { 7-year-olds' choice } & .453 & .343 & .163 \\ \text { Adults' draw } & .699^{\mathrm{b}} & .643^{\mathrm{a}}> & .041 \\ \text { Adults' choice } & .912^{\mathrm{a}} & .631^{\mathrm{a}} & .392^{\mathrm{a}}\end{array}$

Note. Quintic functions marked with $>>$ fit significantly better $(p<.05)$ than both cubic, $F_{S}(2,7)>4.74$, and linear, $F_{S}(4,7)>4.12$, functions. Quintic functions marked with $>$ fit significantly better than only a linear function. Cubic functions marked with $>$ fit significantly better than linear functions, $F_{\mathrm{S}}(2,9)>4.26$.

${ }^{a}$ Curve fits significantly greater than chance, quintic $F_{\mathrm{S}}(5,7)>3.97$, cubic $F_{\mathrm{S}}(3,9)>3.86$, and linear $F_{\mathrm{S}}(1,11)>4.84$.

${ }^{\mathrm{b}}$ Curve fit marginally better than chance, $F(5,7)=3.25, p=.078$.
Percentage of correct responses on choice trials. In addition to assessing the magnitude of error on choice trials, we evaluated the frequency of correct choices. A $t$ test comparing the mean percentage of correct responses across the two age groups revealed a significant difference, $t(58)=-5.90, p<.001$. Adults were correct more often on choice trials $(\mathrm{M}=56.9 \%)$ than children were $(M=30.0 \%)$. Additionally, $t$ tests comparing the performance of each group to chance $(20 \%)$ indicated that both age groups performed at levels significantly above chance: children, $t(29)=3.78$, $p<.01$; adults, $t(29)=9.93, p<.001$.

\section{Discussion}

As in Huttenlocher et al.'s (1994) study, both children's and adults' draw responses were biased away from the edges and the midline of the rectangle. This sinusoidal pattern is consistent with both the DFT and CA models and suggests that draw responses for both ages are driven by the same underlying process (see also Spencer \& Hund, 2003). By contrast, choice responses did not consistently follow this pattern. There were significantly smaller errors on the choice task for both age groups at locations near the inflection points of the sinusoidal draw pattern. More striking, the 7-year-olds' choice responses were not significantly fit by either quintic or cubic functions. Two factors might explain these differences across response types and age groups.

The first reason why children and adults might rely less on geometric category information in the choice task is because there is a bias to use nonspatial information when a verbal response is required. Many verbal statements about objects in the local surround emphasize nonspatial information such as object features. For young children who are still mastering language, it may be less apparent that geometric category information is useful in a verbal response task. Consequently, they may rely primarily on their metric memory when selecting from the set of colored choices. Data from a study by Plumert and Nichols-Whitehead (1996) are consistent with this view. When asked to give directions about how to find a hidden object in a dollhouse, 3- and 4-year-olds were more likely to offer information about the features of a nearby object (e.g., the shoe is hidden under the hat with the blue ribbon) rather than information about locations (e.g., the shoe is hidden under the hat next to the rocking chair). Adults, by contrast, tended to refer to both object features and spatial relations. This is consistent with results from the present study in which children showed little evidence of geometric bias in the choice task whereas adults showed a sinusoidal pattern that was weaker than in the draw task.

A second reason why both age groups showed smaller errors on the choice task is that the added spatial structure provided by the choice dots helped participants generate more accurate responses. Consider, for instance, how one might account for choice responses by using the DFT. According to this model, activation in SWM drifts away from reference axes during delays, and recall

\footnotetext{
2 Because Huttenlocher et al. (1994, Experiment 6) did not round and truncate their data, we analyzed the continuous draw data to allow for a direct comparison with the results found in this study. Huttenlocher et al. reported that a quintic function provided the best fit to their data $\left(R^{2}=.90\right)$. This fit is comparable to the fit of a quintic function to children's continuous draw data in the present experiment $\left(R^{2}=.83\right)$.
} 
responses are generated by pointing to the location associated with maximal activation. In the choice task, it is possible that people use a similar process - they pick the choice that most closely matches the location associated with maximal activation in working memory. This would produce identical errors across response types provided that there is a choice dot in the spatial location currently represented in working memory. If, however, there were substantial drift in working memory-as occurs near the inflection points of the sinusoidal pattern - it is possible that none of the choices would overlap with the remembered location. In this case, participants might pick the closest match or simply guess. This would result in smaller errors on the choice task provided that the choices clustered close to the target location (i.e., the spatial span was small). On average, this was the case in the present experiment, given that the five choices were separated by $5 \mathrm{~mm}$, and the correct choice was constrained to be the second, third, or fourth dot. We tested competing predictions of these alternative accounts in Experiment 2.

\section{Experiment 2}

During the choice trials of Experiment 1, participants selected from a set of five choices separated by $5 \mathrm{~mm}$. To test the competing predictions of the verbal bias and choice span accounts, we increased the number of choices from five to nine (the choices remained separated by $5 \mathrm{~mm}$ ). Thus, the choices now covered a 40-mm spatial span instead of $20 \mathrm{~mm}$. If participants have a bias against using geometric category information in verbal tasks, this manipulation should have no effect relative to Experiment 1. If, however, the improved performance on choice trials in Experiment 1 was caused by a mismatch between a drifting memory and a nonoverlapping and more accurate choice set, then this manipulation should make choice responses more similar to draw responses.

\section{Method}

Participants. Participants were 30 seven-year-olds $\{M$ age $=7.57$ years, $S D=1.05$ months) and 31 adults $(M$ age $=18.76$ years, $S D=9.89$ months). Data from 2 additional children were excluded, 1 because she did not complete the task, and 1 because his draw responses were not clear enough to be coded. All other participant details were as in Experiment 1.

Apparatus. All details of the apparatus were the same as in Experiment 1 except for the choice response cards. These cards had nine choices (rather than five) presented $5 \mathrm{~mm}$ apart and covering a 40-mm span. To keep the choices verbally identifiable, different colored squares and triangles were used. From left to right, the symbols were a black square, a green triangle, a red square, a blue triangle, an orange square, a black triangle, a green square, a red triangle, and a blue square. Participants were asked to say both the color and the symbol type on the choice trials.

Procedure. All procedural details were the same as in Experiment 1.

Experimental design. As in Experiment 1, the locations used for practice and control trials were randomly selected from locations in between the target locations $(6.25 \mathrm{~mm})$. Targets were presented at only 11 locations - the $25-\mathrm{mm}$ and $175-\mathrm{mm}$ locations were not used because there was not enough room to include nine choices between the target and the edge of the card. We also increased the number of control trials to eight to further reduce the likelihood that participants might adopt a strategy in which they avoided selecting the two end choices. In Experiment 1, 7year-olds' percentage of correct responses on test trials was significantly above chance. This was not the case, however, on control trials (when end choices were correct), $t(29)=0.92, p=.36$, suggesting that the children might have been strategically avoiding the end choices. For comparison, adults performed significantly above chance on both test and control trials, $t(29)=7.12, p<.001$

Method of analysis. Choice responses were coded using the method described in Experiment 1. However, because the choices now covered a greater spatial span, the maximum error was $\pm 35 \mathrm{~mm}$. Continuous draw responses were coded as in Experiment 1. Categorical draw errors were rounded to the nearest $5 \mathrm{~mm}$ and truncated to $\pm 35 \mathrm{~mm}$ (rather than to $\pm 15 \mathrm{~mm}$ as in Experiment 1). Again, statistical comparisons revealed no significant differences between continuous and categorical draw errors. Thus, categorical draw errors were used in all analyses.

\section{Results}

Figure 4 shows mean errors for both response types separately for children (Figure 4A) and adults (Figure 4B). Responses followed the sinusoidal pattern seen in Experiment 1, with small errors at the center of the rectangle $(100 \mathrm{~mm})$ and near the centers of each half $(50 \mathrm{~mm}$ and $150 \mathrm{~mm})$. In contrast to Experiment 1, however, errors on both choice and draw trials showed a clear sinusoidal pattern for both age groups. These data were analyzed using a three-way ANOVA with response type (choice or draw) and location $(37.5-162.5 \mathrm{~mm})$ as within-subject factors and age ( 7 years or adult) as a between-subjects factor. This analysis revealed a significant main effect of location, Wilks's $\Lambda=.34, F(10$, $50)=9.56, p<.001$. There were no significant effects of response type or age. These data strongly suggest that the same processes underlie the generation of both verbal and pointing responses in this task. However, considered together with Experiment 1, it is likely that the details of the response context (e.g., spatial span of choices) matter as well.

To examine this issue directly, we compared the choice and draw responses across experiments. Specifically, for each response type we conducted an ANOVA with location (37.5-162.5 mm) as a within-subject factor and age (7 years or adult) and experiment ( 1 or 2 ) as between-subjects factors. Because we used a subset of data from each experiment, we report only significant effects of experiment. Note that the data from trials to the two extreme target locations in Experiment 1 were excluded from these analyses. The ANOVA examining draw responses across experiments revealed no significant experiment effects. Thus, as expected, draw errors were comparable across experiments. By contrast, the ANOVA comparing choice responses revealed a significant Location $\times$ Experiment interaction, $F(10,1170)=2.24, p<.05$. Simple effects tests comparing choice responses in Experiments 1 and 2 at each location separately revealed a significant effect of experiment at two locations near the inflection points of the sinusoidal patternat $75 \mathrm{~mm}$ (Experiment 1: $M=0.00$, Experiment 2: $M=-3.21$ ), $F(1,119)=5.01, p<.05$, and $137.5 \mathrm{~mm}($ Experiment $1: M=0.92$, Experiment $2: M=3.69), F(1,119)=4.56, p<.05$. At both locations, errors were larger in Experiment 2 than in Experiment 1.

Polynomial curve-fitting. Although the data in Figure 4 show a clear sinusoidal pattern, it was important to verify this statistically. Table 2 shows $R^{2}$ values for the fits of quintic, cubic, and linear functions fit separately for each age and response type as well as significant differences in the fits of these curves. Across all four sets of analyses, quintic and cubic functions provided excel- 
lent fits to the data. Relative to Experiment 1, the polynomial trends in 7-year-olds' draw responses ${ }^{3}$ and adults' choice and draw responses were comparable, although these trends were stronger in the present experiment. Moreover, 7-year-olds showed a strong polynomial trend in their choice responses in the present experiment but not in Experiment 1.

Percentage of correct responses on choice trials. A $t$ test comparing the mean percentage of correct responses across the two age groups revealed a significant difference for children and adults, $t(59)=-6.66, p<.001$. As in Experiment 1 , adults $(M=48.4 \%)$ were correct more often than children $(M=16.4 \%)$. Additionally, $t$ tests comparing the performance of each group to chance performance $(11 \%)$ indicated that both age groups performed at levels significantly above chance: children, $t(29)=2.13, p<.01$; adults, $t(30)=9.23, p<.001$.

\section{Discussion}

The present experiment tested competing predictions of two accounts of the smaller errors on choice trials in Experiment 1.

${ }^{3}$ Again, to compare with the findings of Huttenlocher et al. (1994, Experiment 6), we fit curves to the continuous draw data. The fit of a quintic function to children's continuous draw data in the present experiment was excellent $\left(R^{2}=.96\right)$. This fit was higher than the fit reported by Huttenlocher et al. $\left(R^{2}=.90\right)$.
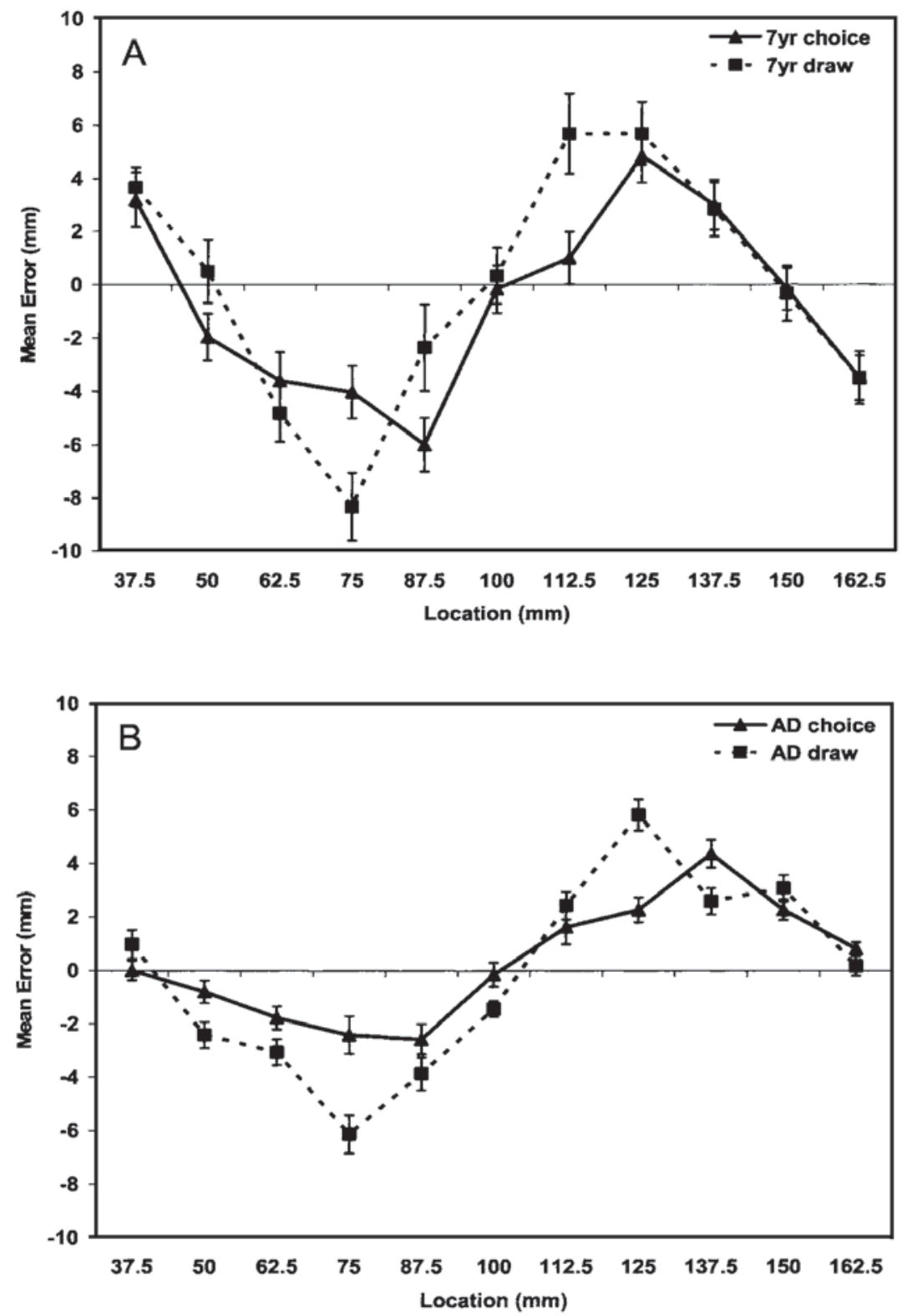

Figure 4. Mean choice and draw errors for (A) 7-year-olds (7yr) and (B) adults (AD) across the 11 target locations in Experiment 2. Positive values indicate rightward errors, and negative values indicate leftward errors. Errors bars reflect plus or minus one standard error. 
Table 2

$R^{2}$ Values of Polynomial Curve Fits in Experiment 2

\begin{tabular}{|c|c|c|c|}
\hline Response & dintic functi & ubic funct & inear function \\
\hline 7-year-olds' draw & $.946^{\mathrm{a}}>>$ & $.800^{\mathrm{a}}>$ & .017 \\
\hline 7-year-olds' choice & $.912^{a}>$ & $.875^{\mathrm{a}}>$ & .025 \\
\hline Adults' draw & $.930^{\mathrm{a}}>$ & $.880^{\mathrm{a}}>$ & .291 \\
\hline Adults' choice & $.959^{\mathrm{a}}>$ & $.892^{\mathrm{a}}>$ & $.444^{\mathrm{a}}$ \\
\hline $\begin{array}{l}\text { Note. Quintic func } \\
\text { than both cubic, } \\
\text { Quintic function } \\
\text { ear function. } \mathrm{Cu} \\
\text { linear functions }\end{array}$ & $\begin{array}{l}\text { ons marked } \\
F_{S}(2,5)>5 \\
\text { marked wi } \\
\text { ic functions } \\
F_{S}(2,7)>4\end{array}$ & $\begin{array}{l}\text { 1 > fit si } \\
\text { and linear, } \\
\text { fit significe } \\
\text { ked with }>\end{array}$ & $\begin{array}{l}\text { cantly better ( } \\
1,5)>5.19 \text {, fu } \\
\text { better than on } \\
\text { ignificantly bet }\end{array}$ \\
\hline $\begin{array}{r}{ }^{\text {a }} \text { Curve fits significa } \\
F_{\mathrm{S}}(3,7)>4.35,\end{array}$ & $\begin{array}{l}\text { tly greater } \\
\text { nd linear } F_{\mathrm{S}}\end{array}$ & $\begin{array}{l}\text { chance, } q \mathrm{q} \\
>5.12 \text {. }\end{array}$ & $F_{\mathrm{S}}(5,5)>5$. \\
\hline
\end{tabular}

Results are not consistent with the proposal that children were biased against using geometric information in verbal tasks. Rather, results support the proposal that the spatial span of the choice set limited participants' choice response errors in the previous experiment. Choice and draw responses in the present experiment did not differ significantly, and responses from both trial types showed a strong and significant sinusoidal pattern. It is important to note that this was the case for both children and adults, suggesting developmental continuity in the processes that underlie performance in this task (see also Spencer \& Hund, 2003).

The present results demonstrate that geometric biases are not unique to sensorimotor responses in spatial recall tasks. This has important implications for the CA and DFT models: It suggests that the processes captured by these models might generalize to verbal responses in recognition tasks. Before these implications were evaluated in detail, however, it was first necessary to address one final empirical issue. To test the choice span account in the present experiment, we increased the number of choices from five to nine. This increased the spatial span of the choice set, but it also increased the number of options from which participants had to choose. Consequently, the increase in geometric bias on choice trials might have resulted from the increased spatial span or a general increase in task difficulty. We tested these possibilities in Experiment 3 .

\section{Experiment 3}

To determine whether the different pattern of results on choice trials across Experiments 1 and 2 was driven by the number of choices or the spatial span of the choices, we used a choice response set with five choices (as in Experiment 1) covering a 40$\mathrm{mm}$ span (as in Experiment 2). If the number of choices caused the differences across experiments, then the results of Experiment 3 should be similar to those of Experiment 1; that is, choice and draw responses should differ significantly. Alternatively, if the spatial span of the choices caused the differences across experiments, then the results of Experiment 3 should be similar to those of Experiment 2; that is, we should find similar patterns of error on both choice and draw trials.

\section{Method}

Participants. Participants were 30 seven-year-olds $(M$ age $=7.55$ years, $S D=0.96$ months $)$ and 30 adults $(M$ age $=19.54$ years, $S D=11.97$ months). All other participant details were the same as in Experiments 1 and 2.

Apparatus. All details of the apparatus were the same as in Experiment 1 except for the choice response cards. These cards had five choices, separated by $10 \mathrm{~mm}$ (rather than $5 \mathrm{~mm}$ ).

Procedure and experimental design. The procedure and design were identical to those used in Experiment 2.

Method of analysis. Choice responses were coded using the method described in Experiment 1. Choices covered the same spatial range as in Experiment 2, but now errors could occur only in $10-\mathrm{mm}$ increments (the distance between two choices). As a result, the maximum error was \pm 30 $\mathrm{mm}$. Continuous draw error was coded the same way as in the previous experiments. Categorical draw error was calculated by rounding the continuous draw errors to $10 \mathrm{~mm}$ and truncating to $\pm 30 \mathrm{~mm}$. As in Experiments 1 and 2, statistical analyses revealed no significant differences between continuous and categorical draw errors, so categorical draw errors were used in all analyses.

\section{Results}

Figure 5 shows mean errors for both response types separately for children (Figure 5A) and adults (Figure 5B). Responses generally followed a sinusoidal pattern as in Experiments 1 and 2, with small errors at the center of the rectangle $(100 \mathrm{~mm})$ and near the centers of each half $(50 \mathrm{~mm}$ and $150 \mathrm{~mm})$. In addition, adults' errors were noticeably smaller than 7-year-olds' errors, as in Experiment 1 but not Experiment 2 .

A three-way ANOVA with response type (choice or draw) and location (37.5-162.5 mm) as within-subject factors and age (7 years or adult) as a between-subjects factor revealed a significant main effect of location, Wilks's $\Lambda=.59, F(10,49)=3.38$, $p<.01$, and a significant Location $\times$ Age interaction, Wilks's $\Lambda$ $=.69, F(10,49)=2.23, p<.05$. As in Experiment 2, there were no significant effects of response type. Adults' errors were generally smaller than children's across the sinusoidal pattern, although tests of simple effects showed a significant effect of age only at one location, $50 \mathrm{~mm}, F(1,58)=4.82, p<.05$. Additional tests of simple effects indicated that there was a significant modulation of error across locations for 7-year-olds, $F(10,290)=3.36, p<.001$, and adults, $F(10,290)=5.40, p<.001$.

The central goal of Experiment 3 was to compare response errors on the choice trials in the present experiment with performance when participants chose from (a) the same number of choices over a small spatial span (Experiment 1) and (b) more choices over the same spatial span (Experiment 2). To examine the effects of spatial span, we compared the choice responses in Experiments 1 and 3 in a three-way ANOVA with location (37.5-162.5 mm) as a within-subject factor and age ( 7 years or adult) and experiment ( 1 or 3 ) as between-subjects factors. We report only significant effects of experiment. The ANOVA revealed a significant Location $\times$ Experiment interaction, $F(10,1160)=2.53, p<.01$. Tests of simple effects indicated that responses at two locations differed significantly across experiments: $75 \mathrm{~mm}, F(1,118)=3.95, p<.05$, and $137.5 \mathrm{~mm}, F(1,118)=4.47, p<.05$. At both of these locations, errors were larger in the present experiment than in Experiment 1. It is important to note that these locations are near the inflection points of the sinusoidal pattern. Next, we examined the influence of the number of choices by comparing the choice responses in Experiments 2 and 3 in a three-way ANOVA. There were no significant effects of experiment. These analyses, in conjunction with 
the absence of significant response type effects in the present experiment, suggest that spatial span — and not the number of choices-is a central contributor to geometric effects in the choice task.

Polynomial curve-fitting. As in the previous experiments, we fit polynomial curves to participants' responses. Table 3 shows $R^{2}$ values for the fits of quintic, cubic, and linear functions fit separately for each age and response type as well as significant differences in the fits of these curves. As in Experiments 1 and 2, the best fit for children's draw data was obtained by a quintic function, though the fit of the cubic function was also quite good. ${ }^{4}$ The same was true of adults' draw responses. For children's choice data, there was no significant difference between the fits of quin- tic, cubic, and linear functions, and none of these functions fit children's responses significantly above chance levels. By contrast, cubic and linear functions fit adults' choice data at levels that were significantly better than chance. This pattern of results is similar to that seen in Experiment 1, raising the possibility that, although the number of choices did not emerge as an important factor on the

${ }^{4}$ Again, to compare with the findings of Huttenlocher et al. (1994, Experiment 6), we fit curves to our continuous draw data. The fit of a quintic function to children's continuous draw data in the present experiment was quite good $\left(R^{2}=.92\right)$ and comparable to results from Huttenlocher et al.
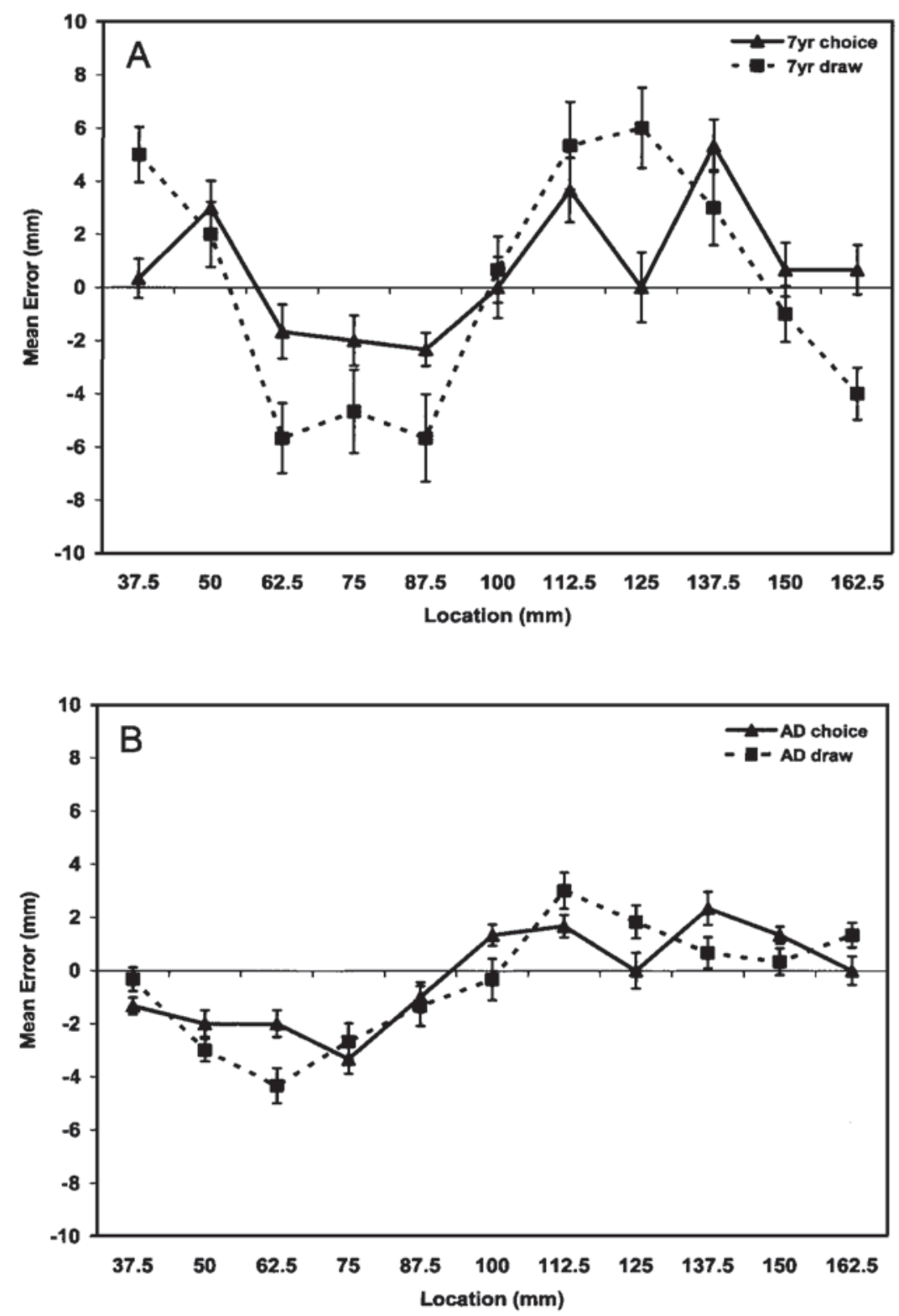

Figure 5. Mean choice and draw errors for (A) 7-year-olds (7yr) and (B) adults (AD) across the 11 target locations in Experiment 3. Positive values indicate rightward errors, and negative values indicate leftward errors. Errors bars reflect plus or minus one standard error. 
Table 3

$R^{2}$ Values of Polynomial Curve Fits in Experiment 3

$\begin{array}{llll}\text { Response } & \text { Quintic function Cubic function Linear function } \\ \text { 7-year-olds' draw } & .957^{\mathrm{a}}>> & .804^{\mathrm{a}}> & .001 \\ \text { 7-year-olds' choice } & .605 & .374 & .084 \\ \text { Adults' draw } & .945^{\mathrm{a}}>> & .741^{\mathrm{a}} & .460 \\ \text { Adults' choice } & .780 & .770^{\mathrm{a}} & .496^{\mathrm{a}}\end{array}$

Note. Quintic functions marked with $>>$ fit significantly better $(p<.05)$ than both cubic, $F_{\mathrm{S}}(2,5)>5.79$, and linear, $F_{\mathrm{S}}(4,5)>5.19$, functions. Quintic functions marked with $>$ fit significantly better than only a linear function. Cubic functions marked with $>$ fit significantly better than linear functions, $F_{\mathrm{S}}(2,7)>4.74$.

${ }^{\text {a }}$ Curve fits significantly greater than chance, quintic $F_{\mathrm{S}}(5,5)>5.05$, cubic $F_{\mathrm{S}}(3,7)>4.35$, and linear $F_{\mathrm{S}}(1,9)>5.12$.

basis of the cross-experiment ANOVAs above, this task factor may play some role in choice responses. Note, however, that children and adults made very small errors to one target location on the choice task-125 mm. Responses to all other targets showed relatively close correspondence across tasks. Given that curve fits take into account only mean responses (thus, we had relatively few degrees of freedom), the reduction in error at $125 \mathrm{~mm}$ likely played a substantial role in the nonsignificant quintic fits. In this context, it is worth noting that the fit of the quintic function approached significance for the children (.605; see Table 3$)$ and was higher than the quintic fit from Experiment 1 (.453).

Percentage of correct responses on choice trials. In a final set of analyses, we examined the percentage of correct responses on choice trials. A $t$ test comparing the mean percentage of correct responses across the two age groups revealed a significant difference, $t(58)=-8.03, p<.001$. As in the previous experiments, adults $(M=73.0 \%)$ were correct more often than children $(M=$ $35.8 \%$ ). Additionally, $t$ tests comparing the performance of each age group to chance $(20 \%)$ indicated that both groups performed at levels significantly above chance: children, $t(29)=4.45, p<$ .001 ; adults, $t(29)=17.66, p<.001$. It is interesting that the percentage of correct responses in the present experiment was noticeably higher than in Experiment 1 (adults: $M=56.9 \%$, children: $M=30.0 \%$ ), despite the fact that both experiments included the same number of choices. To examine this issue directly, we conducted a two-way ANOVA with age (7 years or adult) and experiment ( 1 or 3 ) as between-subjects factors. This analysis revealed a significant main effect of experiment, $F(1,116)=11.29, p<.001$. Participants in Experiment $3(M=54.4 \%)$ were correct significantly more often than in Experiment $1(M=43.5 \%)$. Considered together with mean error on the choice trials, these results indicate how spatial span affects response error in the choice task: Spatial span makes choices more distinctive as evidenced by the higher percentage of correct responses, but there is a cost of this distinctiveness - when participants err, they make large geometric errors that are consistent with biases in the draw task.

\section{Discussion}

This experiment examined whether the spatial span of the choices or the number of choices drove the substantial geometric biases in the choice task of Experiment 2. Results suggest that the spatial span of the choices played a major role in this finding. We saw comparable geometric biases across choice and draw responses in the current experiment. Moreover, cross-experiment comparisons showed a significant increase in error on the choice task at the inflection points of the sinusoidal pattern in this experiment relative to Experiment 1 and no significant differences in error on the choice task across Experiments 2 and 3. These results lend strong support for the choice span account discussed previously. By that view, participants in the choice task select the choice that most closely matches where memory has drifted during the delay. With a larger spatial span, it is more likely that a choice will overlap with where memory has drifted, even when there are relatively few choices from which to pick.

Although our results suggest that the spatial span of the choices is critical, the number of choices does seem to play a role as well. Participants selected the correct choice more often when there were fewer choices (i.e., in Experiments 1 and 3 vs. Experiment 2), particularly when the choices were farther apart (Experiment 3 ). This latter effect was likely caused by the spatial distinctiveness of the choices. It is important to note, however, that we still found evidence of geometric biases on the choice task despite the small number of choices from which to select. One final collection of results contributes to this distinctiveness story: Adults showed significantly smaller errors than 7-year-olds in the present experiment, and they showed a dramatic increase in percentage of correct responses relative to Experiment 1 . This finding suggests that adults capitalized on the distinctiveness of the choices more so than did children. This is consistent with recent proposals that the spatial precision of spatial memory increases systematically over development (e.g., Hund \& Plumert, 2002; Schutte et al., 2003; Spencer \& Hund, 2003). We discuss these findings in greater detail below.

\section{General Discussion}

The purpose of this study was to examine the nature of the memory system that underlies performance when people use spatial memory to form different response types in the presence of different response cues. In particular, we asked whether one type of bias - geometric bias - observed in spatial recall tasks with pointing responses generalizes to a recognition task with a verbal response. If so, it would suggest that the processes that produce geometric biases - processes formalized in models of spatial recallmight be general as well.

Across three experiments, our results clearly show that geometric biases generalize to verbal responses in a recognition task. This was most clearly shown in Experiment 2, in which both children and adults showed robust geometric biases across the draw and choice tasks. However, we also found evidence that the structure of the choice set contributes to the errors participants make in the verbal task. For both age groups, geometric biases in the verbal task were robust in Experiment 2 with a large spatial span and nine choices, weaker in Experiment 3 with a large span and fewer choices, and the weakest in Experiment 1 with a small span and only five choices. These choice dependencies were most dramatic for the 7-year-olds, who showed little evidence of geometric bias in Experiment 1. It is important to note, however, that deviations between choice and draw responses were systematic for both age groups - choice responses were con- 
sistently smaller near the inflection points of the sinusoidal pattern. Spatial span also influenced participants' percentage of correct responses. Percentage of correct responses was higher in Experiment 3 relative to Experiment 1, suggesting that, particularly for adults, the spatial distinc-tiveness of the choices matters.

It is important to note that the choice task we used here differed in multiple ways from the spatial recall task. First, we asked participants to verbally label locations rather than to point - a response difference common in tasks that contrast the "cognitive" and "sensorimotor" systems described above (e.g., Bridgeman et al., 2000; Brungart et al., 2000). Second, the choice task required recognition of the correct location from a set of simultaneously viewable alternatives, rather than recall of the location in an empty rectangle. Although our experiments did not isolate the different contributions of these factors, results demonstrate that geometric biases generalize across these differences. The next challenge is to explain how the different factors may have contributed to both the generalization of bias across tasks and the subtle differences that emerged over development as we manipulated the choice set. We contend that formal models can help meet this challenge by specifying not only whether different factors contributed to performance but also how. Thus, in the sections that follow, we evaluate two central issues. First, given that geometric biases generalized to the choice task, can the CA and DFT models account for our results, and do these models offer insights into the factors that give rise to both the presence and absence of geometric biases across experiments? Second, what are the implications of these results for views that posit two spatial memory systems and discontinuous changes in spatial abilities over development?

\section{Models of Spatial Recall and Geometric Bias in the Choice Task}

The CA model. According to the CA model, geometric biases arise because people weight prototypical information more heavily than less certain fine-grained information after short-term delays (Huttenlocher et al., 1991; Spencer \& Hund, 2002). How might these ideas be applied to the choice task? Central to this question is how people use the choice set, that is, how people combine finegrained, categorical, and choice information to form a response. One possibility is that people use only fine-grained and choice information when generating a response in the verbal task. This idea stems from the proposal discussed earlier that geometric information might be less relevant (or simply used less often) in verbal tasks. Results from Experiment 2 demonstrate that this is not the case in all situations. What might determine when to use categorical information? One likely factor is the certainty of the choice set. With many choices present (Experiment 2), choice-related spatial information would be less certain, and participants might weight prototypical information more heavily. It is important to note, however, that this idea does not appear to capture differences between Experiments 1 and 3. When the choices were more distinctive (Experiment 3), which would presumably lead to greater certainty, we saw stronger geometric biases, not the reverse.

Another possibility is that certainty scales only with the number of choices and not the spatial range. In this case, the weighting of the three types of information would be the same in Experiments 1 and 3 . What, then, might explain the different outcomes in these experiments? It is possible that these outcomes reflect the cost of discretization error in the two situations, that is, error resulting from mapping continuous spatial information onto a discrete response set. For instance, when participants were deciding between two adjacent choices in Experiments 1 and 3, categorical information could occasionally tip the balance in favor of a geometrically biased choice. It is important that the cost of making such a choice would be different in the two experiments - $5 \mathrm{~mm}$ in Experiment 1 versus $10 \mathrm{~mm}$ in Experiment 3. Thus, the same weighting process could produce larger geometric biases in Experiment 3 simply because of the structure of the choice set.

Although these ideas are promising, implementing them in the CA model is not straightforward. For instance, adding a third cue to the model with a particular weighting strength would tend to produce responses in between the options in the choice set. This, of course, never occurs in experiment. There are at least two ways to handle this. First, one could implement a postweighting stage that discretizes the continuous spatial response, mapping space to the most appropriately positioned color in the task space. A second possibility is that weighting occurs in a two-stage process (for a sequential view of spatial language use, see Carlson-Radvansky \& Logan, 1997; Logan \& Sadler, 1996). First, fine-grained and categorical information is weighted on the basis of certainty of the fine-grained information. This would produce a best guess of the remembered location along continuous spatial dimensions. Then, this response could be discretized by combining it with the choice set in a winner-take-all fashion. Both of these seem like reasonable additions to the CA model; however, they highlight the need to formally consider the nature of recognition responses within this modeling framework.

A final question is whether the CA model can capture the developmental changes we observed. As with several other studies in the spatial recall literature, we found that children generally showed larger geometric errors in the draw task than did adults (Huttenlocher et al., 1994; Plumert \& Hund, 2001; Spencer \& Hund, 2003). This finding might indicate that children have a less accurate fine-grained representation of the target location (Hund \& Plumert, 2002; Huttenlocher et al., 1994). Consequently, they weight categorical information more heavily. It is interesting, however, that adults showed a more consistent use of categorical information in the verbal task across experiments. This fits with a recent proposal by Hund and Plumert (2002). These researchers proposed that adults show robust categorical biases in different situations because they have learned over development that the use of categorical information leads to an overall reduction in response error. Similarly, Huttenlocher, Hedges, and Vevea (2000) placed the CA model in a Bayesian framework in which the use of categorical information in adulthood leads to an optimal trade-off between bias and variable error.

The DFT. The starting point for a dynamic field account of the data presented here was discussed previously: Participants might pick the choice that most closely matches the location to which memory has drifted after the delay. Recent ideas proposed by Wilimzig and Schöner $(2005,2006)$ to capture categorical responding with the DFT allow us to make this idea more concrete. Figure 6 shows simulations of a dynamic field model performing the choice task (note that this model is a simplification of our more general theory of SWM described by Spencer and colleagues: Spencer et al., in press; Spencer \& Schöner, 2006). Two fields are displayed within each panel-an SWM field $\left(\mathrm{u}_{\text {swm }}\right)$ and an exci- 
tatory long-term memory field $\left(\mathrm{u}_{\mathrm{ltm}}\right)$. All of the simulations in Figure 6 show performance when the target is presented at a single location, $25 \mathrm{~mm}$ to the right of midline (midline was at Unit 150 and Target 125 was at Unit 225; thus, 5 units along the $x$-axes are equivalent to $1 \mathrm{~mm}$ in the task space). We selected this location because we saw large differences between choice and draw responses near 75 and $125 \mathrm{~mm}$ in the present study. Note that as a final simplification, reference-related long-term memory inputs around the midline axis were captured by static excitatory and inhibitory inputs (in contrast to the dynamic long-term memory inputs in Spencer et al., in press; Spencer \& Schöner, 2006).

As a first step toward describing the full set of simulation results in Figure 6, consider what is depicted in each individual simulation. Each simulation begins with the presentation of a target $25 \mathrm{~mm}$ to the right of midline. This target-related input creates a peak of activation at the target location in the SWM field, which, in turn, increases activation at the associated location in long-term memory. After $4 \mathrm{~s}$ (400 time steps in the model), the target input is turned off, and the peak in SWM drifts away from midline (away from Location 150) during the 10-s delay. Next, the choices are input to the model. This is accompanied by a lowering of the resting level of neurons in SWM, moving the field into "choice" mode. This effectively destroys the target-related activation peak, allowing the choices to structure activation in SWM. Finally, we raised the resting level. As a consequence, the field "chooses" one of the choice inputs; that is, the SWM field forms a peak centered at one of the choices. This is reflected by the activation peak at the end of each simulation.
Given this method for generating a choice response with the DFT, can this model capture results from the present study? The entire set of simulations depicted in Figure 6 provides an initial sketch of the full account. The simulations in the left column depict 7-year-olds' performance across Experiments 1-3, and the simulations in the right column depict adults' performance across the three experiments. The model parameters used for each age group were held constant across the three experiments, and the details of the choice input were changed to match our experimental manipulations: five close choices in Experiment 1, nine close choices in Experiment 2, and five choices far apart in Experiment 3. Across the two age groups, we manipulated the spatial precision of the local excitation/lateral inhibition function that governs neural interactions in SWM. Spencer and colleagues (Schutte et al., 2003; Spencer \& Hund, 2003) have proposed that local excitatory interactions are stronger and more precise later in development with strong, narrow lateral inhibition. The interaction functions used in the simulations are depicted in the inset graphs at the top of Figure 6. All other model parameters were identical across age groups.

Given the changes in neural interaction and the structure of the choice set used here, analyses of the DFT suggest that two factors influenced choice performance in this study. The first factor is the amount of memory drift for the two age groups. As can be seen in Figure 6, the 7-year-old model's memory for the target location drifts dramatically during the memory delay, consistent with the large draw errors reported here and the large spatial recall errors reported in previous studies (see, e.g.. Spencer \& Hund, 2003). By
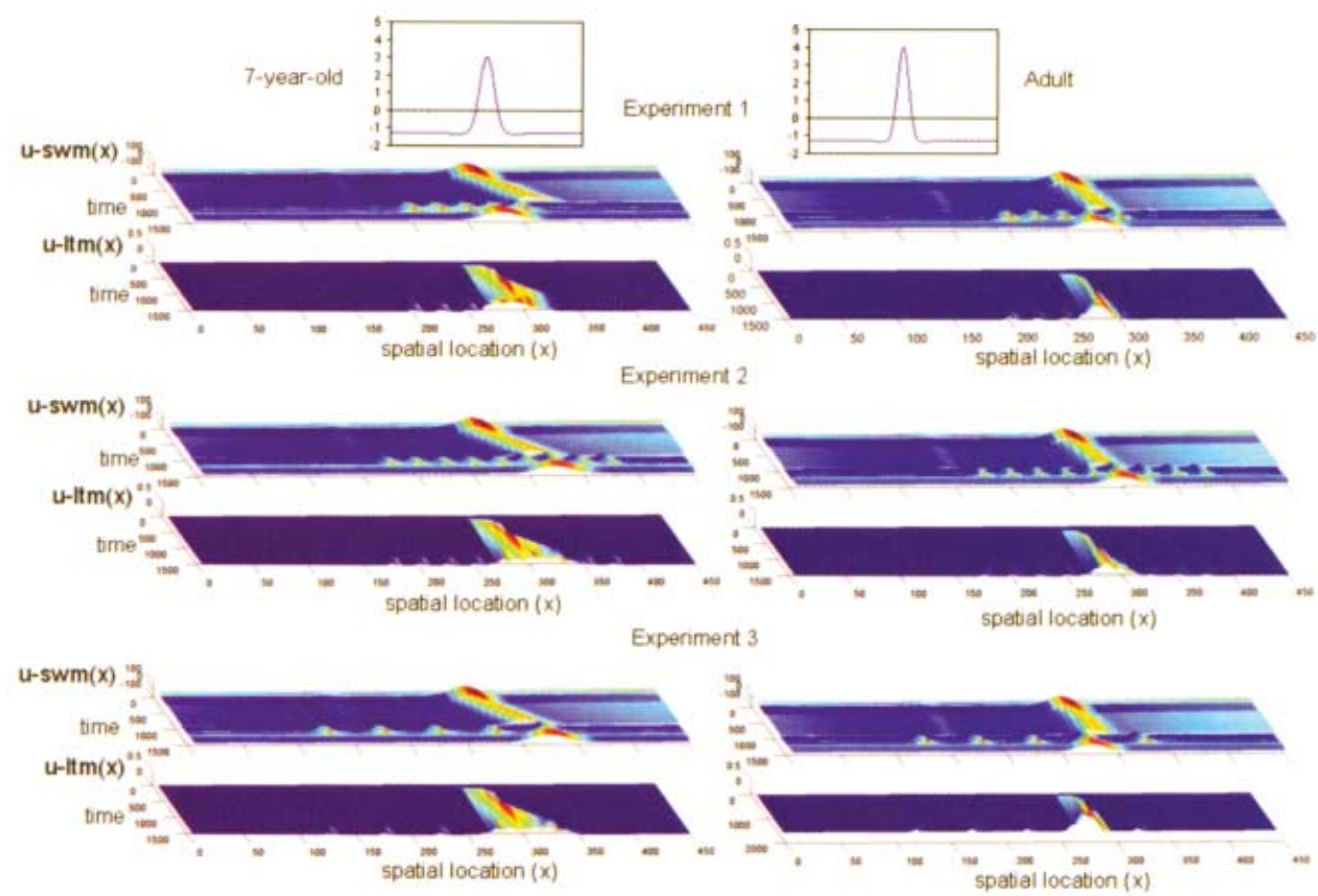

Figure 6. Simulations of the dynamic field theory for a 7-year-old (left column) and an adult (right column) for each experiment. The spatial working memory field, $\mathrm{u}$-swm(x), and the long-term memory field, $\mathrm{u}-1 \mathrm{tm}(\mathrm{x})$, are shown for each simulation. Insets at the top of each column of simulations show the local excitation/lateral inhibition function used for each age group. 
contrast, the adult model's memory drifts much less during the delay, consistent with the significantly smaller errors for the adults in Experiments 1 and 3 and with data from previous studies (see Spencer \& Hund, 2002). The difference in delay-dependent drift in the model results from the difference in neural interaction- activation peaks in the adult model are more precise and more stable. Consequently, they are less influenced by the inhibitory component of the midline input.

Drift in SWM plays a critical role in choice selection because it structures activation in the long-term memory field. It is the activation in this latter field that tips the balance in favor of one choice over another (recall that the target-related peak of activation in SWM is destroyed at the start of the choice interval). As can be seen in Figure 6, the large memory drift of the 7-year-old model produces a broadly distributed pattern of activation in longterm memory, whereas the small memory drift of the adult model produces a more focused activation pattern in long-term memory that is closer to the correct target location.

The second factor that affects choice responses is that the model is biased to select one of the central choices when the choices are close together (Experiments 1 and 2); that is, the model is biased against selecting the outermost choices. Note that the model shows a bias against the outermost choices even though the choices input to the model did not overlap. This occurs because the input provided by the choices blends slightly given the (im)precision of locally excitatory interactions. The blending means the activation on either edge of the inner choice inputs is higher than activation on the outer edges of the outermost choices. This gives a competitive advantage to the inner choices. It is important that this occurs only when the choices are close together, as we demonstrate below. In addition, this bias is stronger for the 7-year-old model given that local excitatory interactions are less precise (see insets in Figure 6).

Taken together, these two factors explain the pattern of simulation results in Figure 6 and provide insights into results of the present study. Consider the performance of the 7-year-old model first. As can be seen in the top left panel of Figure 6, the 7-yearold model selected the correct choice on this trial from Experiment 1 . This occurred because memory drifted beyond the choice set, and the model was biased against selecting the rightmost (the outermost) choice. Note that this is consistent with our empirical findings: Children in Experiment 1 did not perform significantly above chance on the control trials, suggesting that they were biased against selecting an outermost choice. Note also that it is likely that children's memory drifted beyond the choice set relatively frequently in Experiment 1 . As evidence, we conducted a follow-up analysis of the data from Experiment 1, excluding data from locations where memory was unlikely to drift $(37.5,50,100$, 150 , and $167.5 \mathrm{~mm}$ ). Using children's draw responses as an index of where memory drifted for each target location, we found that memory drifted beyond the choice set on $30 \%$ of all choice trials. Children picked the closest choice (the outermost choice) on $28 \%$ of these trials, whereas they picked the second closest choice on $47 \%$ of these trials. Thus, a microanalysis of children's responses is consistent with the behavior of the model.

The second panel in the left column of Figure 6 shows the 7year-old model's performance in a trial from Experiment 2. Here, the model makes a geometrically biased error by selecting the option two choices to the right of the target. As can be seen in the simulation, with nine choices, it is quite likely that there will be a choice in the spatial region to which memory has drifted. This can explain why we found no significant differences between choice and draw responses in this experiment. Finally, in the simulation of a trial from Experiment 3, the 7-year-old model makes a geometrically biased error one choice to the right of the target (see Figure 6). As in Experiment 1, with five choices, there is less of a chance that there will be a choice located in the region to which memory has drifted. This would tend to produce more accurate responses in the choice task. In contrast to Experiment 1, however, the model shows less of a bias against the outermost choices. These two tendencies combined might explain the mixed results in this experiment - we found not only a higher percentage of correct responses for the 7-year-olds but also a greater tendency to show geometric biases in the choice task relative to Experiment 1.

What about the adults? Given the smaller memory drift in the adult model, there is a greater likelihood that there will be a choice that overlaps the location where memory has drifted. Thus, we would expect to see more systematic geometric biases in the choice task for the adults. This is consistent with our empirical results. Nevertheless, as can be seen in the simulation in Figure 6, such geometric biases will be countered in Experiment 1 by the bias against choosing an outermost choice. This might be the source of the smaller geometric biases in the choice task in this experiment. Note that this bias would play the strongest role where memory drift is the largest - at the inflection points of the sinusoidal pattern.

In Experiment 2, we saw robust geometric biases. As can be seen in Figure 6, this is the case with the model as well: The adult model makes a geometrically biased error by selecting the option one choice to the right of the target. It is noteworthy that this bias is smaller than the bias seen with the 7-year-old model. Although this is not consistent with our statistical findings, it is consistent with the smaller errors for the adults in Figure 4. Finally, in Experiment 3, we found that adults picked the correct choice quite often. This is the case with the model as well. Note, however, that the activation peak in the bottom right panel of Figure 6 is close to the outermost target. Thus, in some cases, it is likely that the model will select the geometrically biased choice because of stochastic fluctuations in activation in SWM. As discussed above, this type of digitization error will result in a large error, which could explain the significant geometric bias in Experiment 3 even in the context of a higher percentage of correct responses.

In summary, the simulation results in Figure 6 provide useful insights into the performance of both age groups in the present study. Nevertheless, it is important to note that these insights are qualitative in nature given the lack of quantitative fits. We did not pursue such fits because of practical constraints. To quantitatively model results from this study with the process-oriented approach described here, we would have had to conduct close to 10,000 simulations ( 50 simulations at each of 11 target locations $\times 2$ age groups $\times 3$ selected orientations of the choice set $\times 3$ experiments). Given this unwieldy number of simulations, a more practical approach to the challenge of quantitative simulations would be to conduct more focused empirical studies designed to test specific aspects of the account provided here.

In this context, we return to an issue raised previously: Given that the choice task differed in several ways from the draw task, does the DFT offer insights into how these differences contributed 
to performance? Central to our account are the processes that select a single discrete item from the choice set input to the model. Thus, our account suggests that the presence of several simultaneously available alternatives played a critical role in the task. This predicts that we should see fewer differences between spatial recall performance and performance in a same/different recognition task in which participants must identify whether a single probe item matches a target item after a delay. This is indeed the case (see Simmering, Spencer, \& Schöner, in press; Werner \& Diedrichsen, 2002).

Does this indicate that verbal responses per se (e.g., saying "red" or "same") are unimportant? The answer is no. Indeed, Spencer et al. (in press) recently demonstrated that the same biases reported here arose in a task in which participants had to verbally judge whether a target object was above a reference object. We suspect that the use of spatial prepositions can modify the stability of the SWM processes captured by the DFT. Given that apparently simple changes in the task can lead to a complex pattern of results, the larger message of our model is that a formal understanding of the processes that give rise to performance can make an important contribution to understanding spatial cognition.

\section{Implications for the Two-Memory-Systems Account}

According to the two-spatial-systems view (Milner \& Goodale, 1995), spatial cognition can be partitioned into a sensorimotor system that encodes locations in the service of motor actions and a cognitive system that encodes locations in the service of verbal responses (Bridgeman, 1999; Bridgeman et al., 1981). This distinction is derived from evidence for two cortical pathways for visual information: a ventral stream for picking up information related to object identification and a dorsal stream for handling visual information for action (Lacquaniti et al., 1997; Milner \& Goodale, 1995; Ungerleider \& Mishkin, 1982). It is important that behavioral evidence from experiments with normal populations also supports this distinction. For instance, adults' verbal responses show the Titchner circles illusion (Ebbinghaus illusion): A circle appears to be larger if it is surrounded by smaller circles than if it is surrounded by larger circles. Nevertheless, participants correctly shape their grip when reaching to one of the circles (Aglioti, DeSouza, \& Goodale, 1995), even when reaching to remembered circles, that is, when vision of the hand is occluded during the reach (Haffenden \& Goodale, 1998).

There are many recent behavioral tests of the two-systems view (e.g., Bridgeman et al., 1997, 2000; Haffenden \& Goodale, 1998; Proffitt et al., 1995) and an accompanying set of tests that argue against this distinction (e.g., Franz, Bulthoff, \& Fahle, 2003; Franz, Gegenfurtner, Bülthoff, \& Fahle, 2000; Smeets \& Brenner, 2001; Vishton \& Fabre, 2003; Vishton, Rea, Cutting, \& Nuñez, 1999). The question here is whether the present results have implications for the two-systems proposal. To answer this, we must first consider which memory systems are used in the draw and choice tasks. The drawing task required a sensorimotor response implicating dorsal stream processes, whereas the choice task required a verbal response implicating ventral stream processes. This view is qualified, however, by the type of bias examined-geometric bias. Although geometric bias increases systematically over delay (see Spencer \& Hund, 2002) - a hallmark characteristic of the sensorimotor system (see also Bridge- man et al., 1981; Brungart et al., 2000) — such biases are clearly grounded in an allocentric reference frame. According to Milner and Goodale (1995), this places geometric biases squarely within the ventral stream.

To successfully perform the drawing task, however, people must integrate and coordinate information from both processing streams, taking an allocentric memory of the target location and using it to move the hand in a body-centered reference frame (Milner \& Goodale, 1995). Although results of the present study indicate little about how such coordination takes place, the model used here - the DFT - does. In particular, the simulations presented in Figure 6 show how a single integrated dynamical system can generate both sensorimotor recall responses and verbal choice responses within the same SWM field. Additionally, recent work in autonomous robotics shows how the dynamic field framework can be used to govern the behavior of an autonomous agent as it navigates through the world (Bicho, Mallet, \& Schöner, 2000). It is important to note that this requires the real-time coordination of egocentric and allocentric reference frames, a central challenge to the integration of dorsal and ventral stream processes (for related work, see Pouget, Deneve, \& Duhamel, 2002). Note that applications of the dynamic field framework do not challenge neurophysiological evidence of dorsal and ventral visual pathways; rather, such applications suggest that solutions to the challenge of integration are within reach.

\section{Implications for Spatial Cognitive Development}

Comparisons of children's and adults' responses in the present study revealed a shifting pattern of developmental differences across experiments. Adults' errors were significantly smaller than 7-year-olds' errors in Experiments 1 and 3, but this was not the case in Experiment 2. These results underscore the point that developmental differences must be considered relative to the constraints provided by the task. It is important to emphasize, however, that children and adults remembered locations for different delays in the present study. We did this to make the task more manageable for children yet still reveal robust biases with adults. Clearly, the difference in delays qualifies the lack of significant age-related differences in Experiment 2.

Although we found a complex pattern of developmental results, simulations of the DFT suggest that this model can capture these details through developmental changes in the spatial precision of neural interactions. This adds to the growing list of findings captured by this "spatial precision hypothesis" (see Schutte et al., 2003; Spencer \& Hund, 2003). In particular. Spencer and colleagues have used this same parametric change in the model to account for both quantitative changes in A-not-B-type biases between 2 and 6 years (Schutte et al., 2003) and, more recently, qualitative changes in geometric biases between 3 and 5 years (Schutte, 2004). Note, however, that the current results provide only a limited window onto developmental continuity given that we examined performance at two time points across a broad age range. Nevertheless, it is certainly promising that we were able to capture the performance of 7-year-olds and adults with a single developmental change in the model.

Note also that our results do not address the issue of whether there are important discontinuities in development that occur with the onset of proficient spatial language use (Hermer-Vazquez 
et al., 2001). We examined only one time point in early development, and our choice task included a very constrained use of verbal labeling. This was by design to isolate whether verbal responses in a spatial recognition task would show geometric biases. Clearly, however, it is critical to move beyond this in future extensions of the ideas presented here. For instance. Smith, Samuelson, and Spencer (2006) are currently examining whether concepts of the DFT can help explain how children use space to bind together words and object features. Similarly, results from several recent studies with adults have demonstrated that the DFT can capture novel aspects of adults' use of spatial prepositions such as above (Spencer et al., in press).

\section{Conclusion}

The present study stands at the intersection of three literaturesthe literatures on spatial recall, the two-spatial-systems view, and spatial cognitive development. Our results showing that geometric biases generalize across pointing and verbal response types in both recall and recognition tasks suggest that greater cross-talk among these literatures would be profitable. Indeed, such crosstalk might lead to a different view of the organization of spatial behavior, one that emphasizes the task-specific integration of "sensorimotor" and "cognitive" spatial behaviors rather than the partitioning of behavior into separable subsystems that develop in a discontinuous manner over development. We suggest that the CA and DFT models offer an exciting starting point in this regard, one that might ultimately move these literatures forward toward a more formal, process-oriented future.

\section{References}

Acredolo, L. P. (1985). Coordinating perspectives on infant spatial orientation. In R. Cohen (Ed.), The development of spatial cognition (pp. 115-140). Hillsdale, NJ: Erlbaum.

Aglioti, S., DeSouza, J. F., \& Goodale, M. A. (1995). Size-contrast illusions deceive the eye but not the hand. Current Biology, 5, 679-685.

Bicho, E., Mallet, P., \& Schöner, G. (2000). Target representation on an autonomous vehicle with low-level sensors. International Journal of Robotics Research, 19, 424-447.

Bridgeman, B. (1999). Separate visual representations for perception and for visually guided behavior. In G. Aschersleben, T. Bachmann, \& J. Miisseler (Eds.), Cognitive contributions to the perception of spatial and temporal events. Advances in psychology (pp. 316-327). Amsterdam: Elsevier Science.

Bridgeman, B., Gemmer, A., Forsman, T., \& Huemer, V. (2000). Processing spatial information in the sensorimotor branch of the visual system. Vision Research, 40, 3539-3552.

Bridgeman, B., Kirch, M., \& Sperling, A. (1981). Segregation of cognitive and motor aspects of visual function using induced motion. Perception \& Psychophysics, 29, 336-342.

Bridgeman, B., Peery, S., \& Anand, S. (1997). Interaction of cognitive and sensorimotor maps of visual space. Perception \& Psychophysics, 59, 456-469.

Brungart, D. S., Rabinowitz, W. M., \& Durlach, N. I. (2000). Evaluation of response methods for the localization of nearby objects. Perception \& Psychophysics, 62, 48-65.

Carlson-Radvansky, L. A., \& Logan, G. D. (1997). The influence of reference frame selection on spatial template construction. Journal of Memory and Language, 37, 411-437.

Choi, S., McDonough, L., Bowerman, M., \& Mandler, J. M. (1999). Early sensitivity to language-specific spatial categories in English and Korean. Cognitive Development, 14, 241-268.

Compte, A., Brunei, N., Goldman-Rakic, P. S., \& Wang, X.-J. (2000). Synaptic mechanisms and network dynamics underlying spatial working memory in a cortical network model. Cerebral Cortex, 10, 910-923.

Craton, L. G., Flicker, J., Plumert, J. M., \& Pick, H. (1990). Children's use of frames of reference in communication of spatial location. Child Development, 61, 1528-1543.

Crawford, L. E., Regier, T., \& Huttenlocher, J. (2000). Linguistic and non-linguistic spatial categorization. Cognition, 75, 209-235.

Engebretson, P. H., \& Huttenlocher, J. (1996). Bias in spatial location due to categorization: Comment on Tversky and Schiano. Journal of Experimental Psychology: General, 125, 96-108.

Franz, V. H., Bülthoff, H. H., \& Fahle, M. (2003). Grasp effects of the Ebbinghaus illusion: Obstacle avoidance is not the explanation. Experimental Brain Research, 149, 470-477.

Franz, V. H., Gegenfurtner, K. R., Bülthoff, H. H., \& Fahle, M. (2000). Grasping visual illusions: No evidence for a dissociation between perception and action. Psychological Science, 11, 20-25.

Georgopoulos, A. P., Taira, M., \& Lukashin, A. V. (1993, April 2). Cognitive neurophysiology of the motor cortex. Science, 260, 47-52.

Goldman-Rakic, P. S. (1995). Cellular bases of working memory. Neuron, 14, 477-485.

Haffenden, A. M., \& Goodale, M. A. (1998). The effect of pictorial illusion on prehension and perception. Journal of Cognitive Neuroscience, 10, 122-136.

Hermer, L., \& Spelke, E. S. (1994). A geometric process for spatial reorientation in young children. Nature, 370, 57-59.

Hermer, L., \& Spelke, E. S. (1996). Modularity and development: The case of spatial reorientation. Cognition, 61, 195-232.

Hermer-Vazquez, L., Moffet, A., \& Munkholm, P. (2001). Language, space, and the development of cognitive flexibility in humans: The case of two spatial memory tasks. Cognition, 79, 263-299.

Hermer-Vazquez, L., Spelke, E. S., \& Katsnelson, A. S. (1999). Sources of flexibility in human cognition: Dual-task studies of space and language. Cognitive Psychology, 39, 3-36.

Hespos, S. J., \& Spelke, E. S. (2004). Conceptual precursors to language. Nature, 430, 453-456.

Hund, A. M., \& Plumert, J. M. (2002). Delay-induced bias in children's memory for location. Child Development, 73, 829-840.

Huttenlocher, J., Hedges, L. V., \& Duncan, S. (1991). Categories and particulars: Prototype effects in estimating spatial location. Psychological Review, 98, 352-376.

Huttenlocher, J., Hedges, L. V., \& Vevea, J. L. (2000). Why do categories affect stimulus judgment? Journal of Experimental Psychology: General, 129, 220-241.

Huttenlocher, J., Newcombe, N., \& Sandberg, E. H. (1994). The coding of spatial location in young children. Cognitive Psychology, 27, 115-147.

Jackendoff, R. (1996). The architecture of the linguistic-spatial interface. In P. Bloom, M. A. Peterson, L. Nadel, \& M. F. Garret (Eds.), Language and space (pp. 1-30). Cambridge, MA: MIT Press.

Lacquaniti, F., Perani, D., Guigon, E., Bettinardi, V., Carrozzo, M., Grassi, F., et al. (1997). Visuomotor transformations for reaching to memorized targets: A PET study. NeuroImage, 5, 129-146.

Laeng, B., Peters, M., \& McCabe, B. (1998). Memory for locations within regions: Spatial biases and visual hemifield differences. Memory \& Cognition, 26, 97-107.

Learmonth, A. E., Newcombe, N., \& Huttenlocher, J. (2001). Toddlers' use of metric information and landmarks to reorient. Journal of Experimental Child Psychology, 80, 225-244.

Logan, G. D., \& Sadler, D. D. (1996). A computational analysis of the apprehension of spatial relations. In P. Bloom, M. A. Peterson, L. Nadel, \& M. F. Garrett (Eds.), Language and space (pp. 493-529). Cambridge, MA: MIT Press. 
Mclntyre, J., Stratta, F., \& Lacquaniti, F. (1998). Short-term memory for reaching to visual targets: Psychophysical evidence for bodycentered reference frames. Journal of Neuroscience, 18, 8423-8435.

Milner, D., \& Goodale, M. A. (1995). The visual brain in action. Oxford, England: Oxford University Press.

Nelson, T. O., \& Chaiklin, S. (1980). Immediate memory for spatial location. Journal of Experimental Psychology: Human Learning and Memory, 6, 529-545.

Newcombe, N., Huttenlocher, J., \& Learmonth, A. E. (1999). Infants' coding of location in continuous space. Infant Behavior \& Development, 22, 483-510. Piaget, J. (1954). The construction of reality in the child. New York: Basic Books.

Plumert, J. M., Ewert, K., \& Spear, S. J. (1995). The early development of children's communication about nested spatial relations. Child Development, 66, 959-969.

Plumert, J. M., \& Hund, A. M. (2001). The development of location memory: What role do spatial prototypes play? Child Development, 72, 370-384.

Plumert, J. M., \& Nichols-Whitehead, P. (1996). Parental scaffolding of young children's spatial communication. Developmental Psychology, 32, 523-532.

Pouget, A., Deneve, S., \& Duhamel, J. R. (2002). A computational perspective on the neural basis of multisensory spatial representations. Nature Reviews Neuroscience, 3, 741-747.

Proffitt, D. R., Bhalla, M., Gossweiler, R., \& Midgett, J. (1995). Perceiving geographical slant. Psychonomic Bulletin \& Review, 2, 409-428.

Renart, A., Song, P., \& Wang, X.-J. (2003). Robust spatial working memory through homeostatic synaptic scaling in heterogeneous cortical networks. Neuron, 38, 473-485.

Sandberg, E. H., Huttenlocher, J., \& Newcombe, N. (1996). The development of hierarchical representation of two-dimensional space. Child Development, 67, 721-739.

Schiano, D. J., \& Tversky, B. (1992). Structure and strategy in encoding simplified graphs. Memory \& Cognition, 20, 12-20.

Schutte, A. R. (2004). A developmental transition in spatial working memory. Unpublished doctoral dissertation, University of Iowa.

Schutte, A. R., \& Spencer, J. P. (2002). Generalizing the dynamic field theory of the A-not-B error beyond infancy: Three-year-olds' delay- and experience-dependent location memory biases. Child Development, 73, 377-404.

Schutte, A. R., Spencer, J. P., \& Schöner, G. (2003). Testing the dynamic field theory: Working memory for locations becomes more spatially precise over development. Child Development, 74, 1393-1417.

Simmering, V. R., Spencer, J. P., \& Schöner, G. (in press). Referencerelated inhibition produces enhanced position discrimination near axes of symmetry. Perception \& Psychophysics.

Smeets, J. B. J., \& Brenner, E. (2001). Independent movements of the digits in grasping. Experimental Brain Research, 139, 92-100.

Smith, L. B., Samuelson, L. K., \& Spencer, J. P. (2006). The role of space in binding names to things. Manuscript in preparation.

Smith, L. B., Thelen, E., Titzer, R., \& McLin, D. (1999). Knowing in the context of acting: The task dynamics of the A-not-B error. Psychological Review, 106, 235-260.

Soechting, J. F., \& Flanders, M. (1989). Sensorimotor representations for pointing to targets in three-dimensional space. Journal of Neurophysiology, 62, 582-594.

Spencer, J. P., \& Hund, A. M. (2002). Prototypes and particulars: Geometric and experience-dependent spatial categories. Journal of Experimental Psychology: General, 131, 16-37.

Spencer, J. P., \& Hund, A. M. (2003). Developmental continuity in the processes that underlie spatial recall. Cognitive Psychology, 47, 432-480.

Spencer, J. P., Lipinski, J., \& Samuelson, L. K. (in press). It's in the eye of the beholder: Spatial language and spatial memory use the same perceptual reference frames. In L. B. Smith, M. Gasser, \& K. Mix (Eds.), The spatial foundations of language. Oxford, England: Oxford University Press.

Spencer, J. P., \& Schöner, G. (2003). Bridging the representational gap in the dynamical systems approach to development. Developmental Science, 6, 392-412.

Spencer, J. P., \& Schöner, G. (2006). A dynamic field theory of spatial working memory. Manuscript in preparation.

Spencer, J. P., \& Schutte, A. R. (2004). Unifying representations and responses: Perseverative biases arise from a single behavioral system. Psychological Science, 15, 187-193.

Spencer, J. P., Smith, L. B., \& Thelen, E. (2001). Tests of a dynamic systems account of the A-not-B error: The influence of prior experience on the spatial memory abilities of 2-year-olds. Child Development, 72, 1327-1346.

Talmy, L. (1983). How language structures space. New York: Plenum Press.

Thelen, E., Schöner, G., Scheier, C., \& Smith, L. B. (2001). The dynamics of embodiment: A dynamic field theory of infant perseverative reaching errors. Behavioral and Brain Sciences, 24, 1-86.

Tversky, B., \& Schiano, D. J. (1989). Perceptual and conceptual factors in distortions in memory for graphs and maps. Journal of EXperimental Psychology: General, 118, 387-398.

Ungerleider, L. G., \& Mishkin, M. (1982). Two cortical visual systems. In D. J. Ingle, M. A. Goodale, \& R. J. W. Mansfield (Eds.), Analysis of visual behavior (pp. 549-586). Cambridge, MA: MIT Press.

Vishton, P. M., \& Fabre, E. (2003). Effects of the Ebbinghaus illusion on different behaviors: One- and two-handed grasping; one- and two-handed manual estimation; metric and comparative judgment. Spatial Vision, 16, 377-392.

Vishton, P. M., Rea, J. G., Cutting, J. V., \& Nuñez, L. N. (1999). Comparing effects of the horizontal-vertical illusion on grip scaling and judgment: Relative versus absolute, not perception versus action. Journal of Experimental Psychology: Human Perception and Performance, 25, 1659-1672.

Werner, S., \& Diedrichsen, J. (2002). The time course of spatial memory distortions. Memory \& Cognition, 30, 718-730.

Wilimzig, C., \& Schöner, G. (2005, July). The emergence of stimulusresponse associations from neural activation fields: Dynamic field theory. Poster session presented at the 27th Annual Conference of the Cognitive Science Society, Stresa, Italy.

Wilimzig, C., \& Schöner, G. (2006). Where do the choices get into the nervous system? A dynamic field theory of categorical and continuous responding. Manuscript in preparation. 